Research Article

\title{
The Efficacy and Safety of Traditional Chinese Medicine Tonifying-Shen (Kidney) Principle for Primary Osteoporosis: A Systematic Review and Meta-Analysis of Randomized Controlled Trials
}

\author{
Junquan Liang, ${ }^{1}$ Fengyi Wang, Jiajia Huang, ${ }^{1}$ Yunxiang Xu $\mathbb{D},{ }^{1}$ and Guizhen Chen $\mathbb{D}^{2}$ \\ ${ }^{1}$ Clinical Medical School of Acupuncture, Moxibustion and Rehabilitation, Guangzhou University of Chinese Medicine, \\ Guangzhou 510405, Guangdong, China \\ ${ }^{2}$ The Bao'an District TCM Hospital, The Affiliated Hospital of Guangzhou University of Chinese Medicine, \\ Guangzhou University of Chinese Medicine, Shenzhen 518101, Guangdong, China
}

Correspondence should be addressed to Yunxiang Xu; xuyx1968@163.com and Guizhen Chen; cgzhen2000@163.com

Received 8 April 2020; Revised 30 August 2020; Accepted 16 September 2020; Published 6 October 2020

Academic Editor: George B. Lenon

Copyright ( $\odot 2020$ Junquan Liang et al. This is an open access article distributed under the Creative Commons Attribution License, which permits unrestricted use, distribution, and reproduction in any medium, provided the original work is properly cited.

\begin{abstract}
Objective. This study aimed to appraise the efficacy and safety of the tonifying-Shen (kidney) principle (TS (TK) principle) for primary osteoporosis (POP). Methods. Randomized controlled clinical trials (RCTs) using the TS (TK) principle for POP were searched from eight electronic databases to search for relevant literature that was published from the initiation to September 2019. Two reviewers performed study selection, data extraction, data synthesis, and quality assessment independently. Review Manager 5.3 software was used to assess the risk of bias and conduct the data synthesis. We assessed the quality of evidence for outcomes by using the Grading of Recommendations Assessment, Development, and Evaluation (GRADE) system. Results. Thirty-six studies with 3617 participants were included. Meta-analysis showed a consistently superior effect of the TS (TK) principle combined with conventional Western medicine $(\mathrm{CWM})$ in terms of total effectiveness rates $(\mathrm{RR}=1.28 ; 95 \% \mathrm{CI}(1.23,1.33) ; P<0.00001)$, $\mathrm{BMD}$ of the lumbar spine $(\mathrm{SMD}=0.71 ; 95 \% \mathrm{CI}(0.47,0.95) ; P<0.00001)$ and proximal femur $(\mathrm{SMD}=0.94 ; 95 \% \mathrm{CI}(0.49,1.38)$; $P<0.00001)$, TCM symptom integral (SMD $=-1.23 ; 95 \%$ CI $(-1.43,-1.02) ; P<0.00001)$, and VAS scores $(\mathrm{SMD}=-3.88 ; 95 \% \mathrm{CI}$ $(-5.29,-2.46) ; P<0.00001)$, when compared to using CWM alone and with significant differences. Besides, in respect of adverse effects, it showed no significant statistical difference between the experimental and control groups, $\mathrm{RR}=0.99$ and $95 \% \mathrm{CI}(0.65$, 1.51), $P=0.97$. Conclusion. Our meta-analysis provides promising evidence to suggest that using the TS (TK) principle combined with CWM for POP is more effective than using CWM alone. Also, both of them are safe and reliable for POP.
\end{abstract}

\section{Introduction}

Primary osteoporosis (POP), also called "a silent disease," is one of the pandemic public health issues that seriously threaten people's health all over the world [1]. Theoretically, POP and secondary osteoporosis belong to the field of osteoporosis (OP). POP includes two major types: postmenopausal osteoporosis (PMOP) and age-related or senile osteoporosis [2]. POP is characterized by decreased bone mass and degenerated bone microstructure, which contributes to a high risk of bone fragility and fracture $[3,4]$. It is estimated that the number of individuals aged 50 years or over at high risk of osteoporotic fracture worldwide was at 158 million in 2010 and is set to double by 2040 [5]. In China, because of the largest population and the increasing proportion of elderly people, osteoporosis has become a severe challenge to the Chinese family, society, and government $[6,7]$. Therefore, an effective prevention and treatment method is urgently needed for POP. Management of POP includes nonpharmacologic treatment and pharmacologic treatment. Conventional Western medicines (CWM) including antiresorptive or anabolic are widely used in the 
treatment of POP [8-10]. However, due to adverse effects and risk of cancer, the use of CWM as long-term treatment is limited [11].

It is well documented that traditional Chinese medicine (TCM) is commonly combined with pharmacotherapy for POP in China [12-14]. In the theory of TCM, there is no particular disease named POP. According to the clinical symptoms, POP belongs to the TCM category of "Guwei (flaccidity of bones)," "Guku (dryness of bones)," "Guji (disease of bones)," "Gukong (depletion of bones), and Gubi (impediment of bones)" [15]. "Shen (kidney) dominating the bone" is the most fundamental theory in TCM. Shen essence (kidney essence) is closely related to bone physiology and pathology. The sufficient Shen essence can keep the bone healthy and strong. On the contrary, the deficiency of Shen essence will lead to a series of bone-related symptoms. Besides, "liver controlling tendon," is involved in bone health. Also, the spleen and stomach are considered as the postnatal foundations of organisms and are the material sources of bone growth. Therefore, the tonifying-Shen (kidney) principle (TS (TK) principle) mainly includes bushen huoxue, bushen zhuanggu, bushen jianpi, and buyi ganshen, which are widely used in combination with other TCM therapies or CWM for the treatment of POP. And, this significant principle has its essential meaning in curing POP [16].

In recent years, numerous meta-analyses were carried out to investigate the efficacy of acupuncture or Chinese herbal medicine for POP [17]. However, there was still no sufficient evidence to draw definitive conclusions as most studies were not comparative analysis aiming at the effect of the TS (TK) principle for POP. Besides, most of the metaanalyses did not explore the safety of particular interventions. Therefore, the purpose of this study was to systematically identify available randomized clinical trials (RCTs) using the TS (TK) principle combined with CWM for POP to appraise its efficacy and safety.

\section{Materials and Methods}

2.1. Study Registration. The study has been registered in PROSPERO (registration number: CRD42020151768). The review reporting was conducted in compliance with the preferred reporting items for systematic reviews and metaanalyses (PRISMA) statement guidelines.

\subsection{Study Design}

\subsubsection{Inclusion Criteria}

(1) Types of Participants and Interventions. All RCTs that were reporting the application of the TS (TK) principle combined with CWM for POP were included. The interventions of these studies must include the TS (TK) principle in the experimental group. Studies must be published in English or Chinese language. TS (TK) principle mainly includes bushen huoxue, bushen zhuanggu, bushen jianpi, and buyi ganshen. Specific treatment methods include traditional Chinese herbal medicine, acupuncture and moxibustion combined with traditional Chinese herbal medicine, acupoint catgut embedding, and acupoint injection. POP patients were included. There is no restriction on gender, race, ethnicity, or nation. Patients in the treatment group were given the TS (TK) principle combined with CWM, while patients in the control group were given CWM alone. The dosages and courses were not limited in our studies.

(2) Types of Outcome Measures. The primary outcomes included effectiveness rate and bone mineral density (BMD) of the lumbar spine and proximal femur (femoral neck or total hip). The secondary outcomes consisted of VAS scores and TCM symptom integral. Besides, adverse events were also assessed as a safety measurement.

2.2.2. Exclusion Criteria. Studies with the following characteristics were excluded: irrelevant to TS (TK) principle studies; studies without consistent diagnostic criteria or relevant outcome indicators; non-English or Chinese-language articles; duplicate reports or the data cannot be extracted; case reports, animal experiences, qualitative studies, comments, or review articles.

2.3. Literature Search Strategy. We searched four international electronic databases (PubMed, Cochrane Library, EMBASE, and Web of Science) and four Chinese electronic databases (CNKI, VIP, Wanfang, and CBM) from their initiation to September 2019 to collect for relevant literature. The literature search was constructed around search terms for TS (TK) principle, POP, and randomized controlled trials and adapted for each database as necessary. The references of the included studies were also screened for further material for inclusion. The detailed search strategy for PubMed is in Table 1. Search strategies were also used for other electronic databases.

2.4. Study Selection and Data Extraction. As a first step in the data handling process, titles and abstracts of all studies retrieved by the search strategies were screened for relevance, and all those that were clearly irrelevant have been discarded.

As a second step, two review team members (Junquan Liang and Fengyi Wang) independently assessed the eligibility of the studies by using the predefined inclusion and exclusion criteria. Besides, for the studies that meet the inclusion criteria, the whole article was read by reviewers to ensure that the entire study met the criteria and was prepared to extract relevant information. The disagreements on whether including a specific study or not were resolved by discussion between the reviewers. The lacking information was requested by contacting the writer of the original article.

The information extracted by the two independent review team members included the following: study setting, population study, participant demographics and baseline characteristics, details of the intervention and control conditions, study methodology, outcomes and treatment 
periods, information for the assessment of the risk of bias. The discrepancies were identified and resolved through discussion (with a third author where necessary). Missing data were requested from the study authors.

2.5. Risk of Bias Assessment. There were two reviewers involved in the quality assessment process, and any major disagreements were resolved by discussion to define the final set of included studies.

Two independent reviewers assessed the risk of bias by considering the following characteristics: randomization sequence generation, treatment allocation concealment, blinding method, completeness of outcome data, selective outcome reporting, and other sources of bias. Besides, the Cochrane Collaboration's risk of bias assessment tool was used to assess the quality of the individual included studies.

2.6. Data Synthesis. Review Manager 5.3 software was used to carry out the quantitative synthesis. Mean difference (MD) or standardized mean difference (SMD) was used for continuous data. Risk ratio (RR) was used for the analysis of dichotomous data. Both were given a $95 \%$ confidence interval $(\mathrm{CI})$. In the case of homogeneous data $\left(I^{2} \leq 50 \%\right.$, $P>0.10$ ), the fixed-effect model was adopted for the metaanalysis. Otherwise, the sources of heterogeneity were further analyzed. After excluding the influence of marked clinical heterogeneity, a random-effect model was adopted to perform the meta-analysis. Sensitivity and bias risk analyses were also performed.

2.6.1. Analysis of Subgroups. We performed some planned subgroup analysis: different specific therapies (bushen huoxue, bushen zhuanggu, bushen jianpi, and buyi ganshen) included in the TS (TK) principle, different kinds of treatment methods (traditional Chinese herbal medicine, acupuncture and moxibustion combined with traditional Chinese herbal medicine, acupoint catgut embedding, and acupoint injection), different parts of BMD examination (lumbar spine and proximal femur (femoral neck or total hip)), and different treatment periods of the TS (TK) principle ( $\leq 3$ months, 3-6 months, and $>6$ months).

2.6.2. Sensitivity Analysis and Reporting Bias Analysis. Sensitivity analysis was carried out to identify the robustness and stability of pooled outcome results by removing the lowquality studies. We have performed a funnel plot of the primary outcome (effectiveness rates of different treatment methods included in the tonifying-Shen (kidney) principle) to evaluate the reporting bias.

2.7. Quality of Evidence. We assessed the quality of evidence for outcomes by using the Grading of Recommendations Assessment, Development, and Evaluation (GRADE) system [18].

\section{Results}

3.1. Study Description and Participants. We obtained 390 relevant studies through preliminary searches. After multiple filtering steps, 36 RCTs with a total of 3617 participants were ultimately included in this systematic review. The flowchart of all study selection procedures is shown in Figure 1.

The 36 included studies involved 3617 participants. Among these studies, apart from combined with CWM, 23 studies reported using TCM herbal medicine [19-41], 5 studies reported using acupuncture and moxibustion combined with traditional Chinese herbal medicine [42-46], 9 studies reported using acupoint catgut embedding [25, 26, 40, 47-52], and 2 studies reported using acupoint injection [53, 54]. Besides, 3 studies divided their experimental group into two groups, respectively (acupoint catgut embedding group and TCM herbal medicine group) $[25,26,40]$. The detailed characteristics of the included studies are shown in Table 2.

3.2. Risk of Bias Assessment. We used the Cochrane Collaboration's risk of bias assessment tool to assess the quality of the included studies. Firstly, all studies reported the method of randomization, and 30 studies described the method of generating a randomization number table [19-30, 32, 34, 35, 37-42, 44, 46, 48-54]. The remaining methods to achieve the sequence generation process include the following: drawing opaque envelope randomly [43], using Doll's clinical case random table [47], drawing of lots, $[36,45]$, and tossing coins $[31,33]$. Secondly, there were only 3 studies which achieved allocation concealment $[19,20,43]$. Thirdly, 3 studies were assessed as appropriate doubleblinding of participants and provided detailed information for double-blinding during treatment as well as an outcome assessment $[19,20,43]$. None of the studies reported any incomplete outcome data (Table 3 and Figure 2).

\subsection{Meta-Analysis}

3.3.1. Effectiveness Rates of Different Treatment Methods Included in TS (TK) Principle. Twenty-five RCTs reported effectiveness rates of different treatment methods included in the TS (TK) principle [21-23, 25, 27, 28, 30, 31, 33, 34, 36-42, 44-50, 53]. Sixteen RCTs reported effectiveness rates of TCM herbal medicine $[21-23,25,27,28,30,31,33,34,36-41]$, and there was low statistical heterogeneity among studies $\left(\mathrm{chi}^{2}=19.09\right.$, $P=0.21 ; I^{2}=21 \%$ ). Therefore, the fixed-effect model was applied to calculate the combined RR and $95 \% \mathrm{CI}$ as 1.24 $(1.19,1.30), P<0.00001$, indicating a statistically significant difference between TCM herbal medicine combined with CWM and CWM alone. This result suggests that TCM herbal medicine combined with CWM in the treatment of POP can significantly improve clinical efficacy when compared with using CWM alone. Three studies reported effectiveness rates of acupuncture and moxibustion combined with TCM herbal medicine $[42,44,45]$. The result showed 


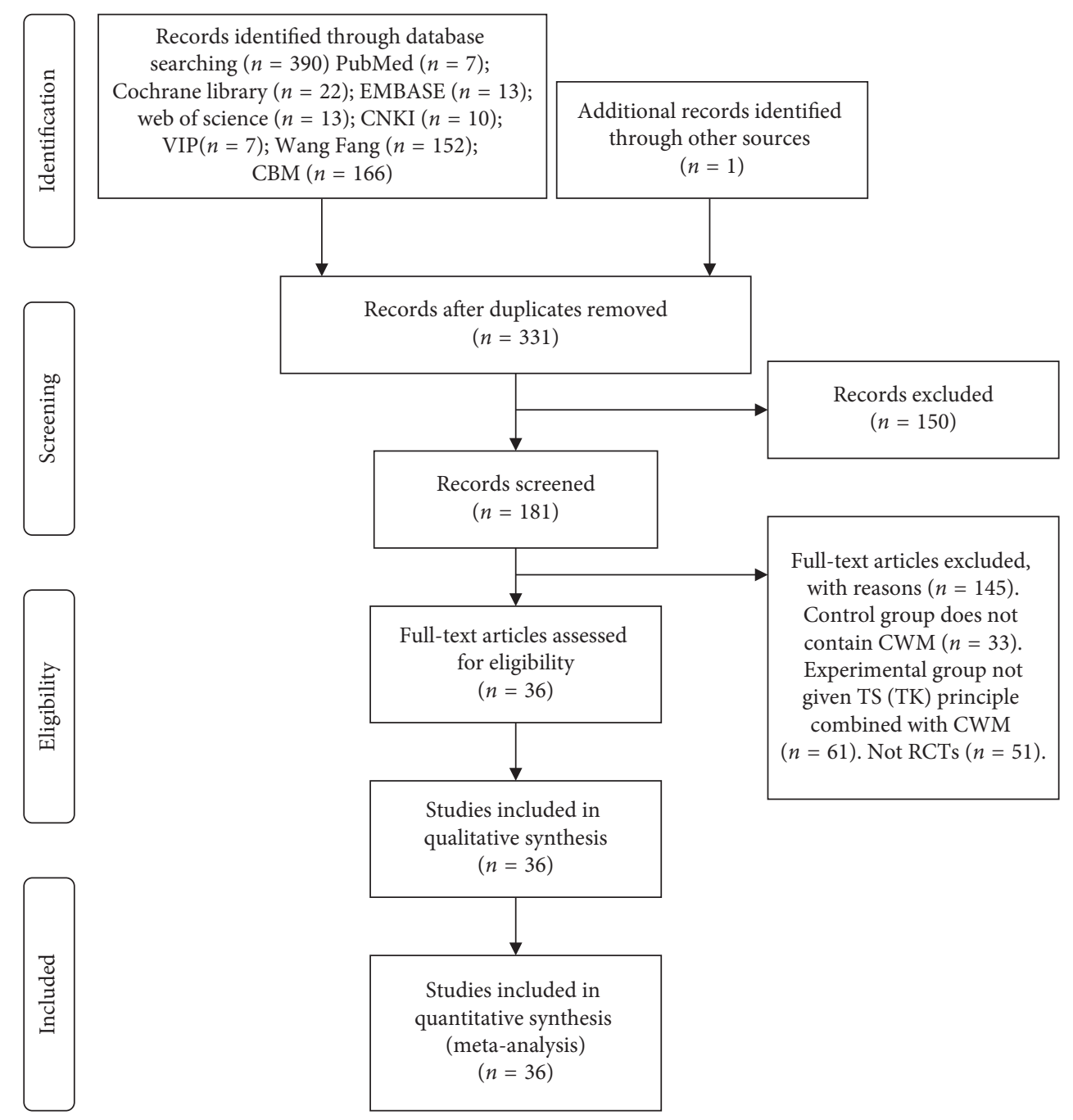

Figure 1: The flowchart of the selection procedure.

that there was no statistical heterogeneity among studies $\left(\mathrm{chi}^{2}=3.94, P=0.14 ; I^{2}=49 \%\right.$ ), so we adopted a fixed-effect model to calculate the combined RR and 95\% CI as 1.34 (1.19, 1.51), $P<0.00001$, indicating a statistically significant difference between the experimental group and the control group. This result suggests that, in respect of effectiveness rates, using acupuncture and moxibustion combined with TCM herbal medicine plus CWM for POP was better than using CWM alone. There are 6 studies which reported effectiveness rates of acupoint catgut embedding $[25,40,47-50]$. The heterogeneity was not detected among studies $\left(\mathrm{chi}^{2}=4.53, P=0.48 ; I^{2}=0 \%\right)$, so a fixed-effect model was used to calculate the combined RR and 95\% CI as 1.42 (1.27, 1.58), $P<0.00001$, indicating a statistically significant difference between acupoint catgut embedding combined with the CWM group and the CWM alone group. This result shows that the effectiveness rates of catgut embedding combined with CWM for POP were better than using CWM alone. There is only 1 study which reported effectiveness rates of acupoint injection [53]. The combined RR and 95\% CI was $1.25(1.05,1.48), P<0.00001$, indicating the difference between acupoint injection combined with the CWM group and the CWM alone group. Owing to the small sample size, this result would show that the effectiveness rates of acupoint injection combined with CWM for POP were better than using CWM alone. All in all, the pooled data showed that different treatment methods included in the TS (TK) principle combined with CWM were more effective than using CWM alone in improving effectiveness rates, with significant differences $(\mathrm{RR}=1.28 ; 95 \%$ CI $(1.23,1.33) ; \quad P<0.00001)$ (Figure 3(a)).

3.3.2. Effectiveness Rates of Different Specific Therapies Included in TCM Herbal Medicine. There were 16 studies which reported effectiveness rates of different specific therapies included in TCM herbal medicine $[21-23,25,27,28,30,31,33,34,36-41]$. Five studies reported the effectiveness rates of bushen huoxue therapy [23, 36-39], three studies reported the effectiveness rates of bushen zhuanggu therapy $[27,31,33]$, three studies reported the effectiveness rates of bushen jianpi therapy [22, 28, 30], and five studies reported the effectiveness rates of buyi ganshen therapy $[21,25,34,40,41]$. The heterogeneity 


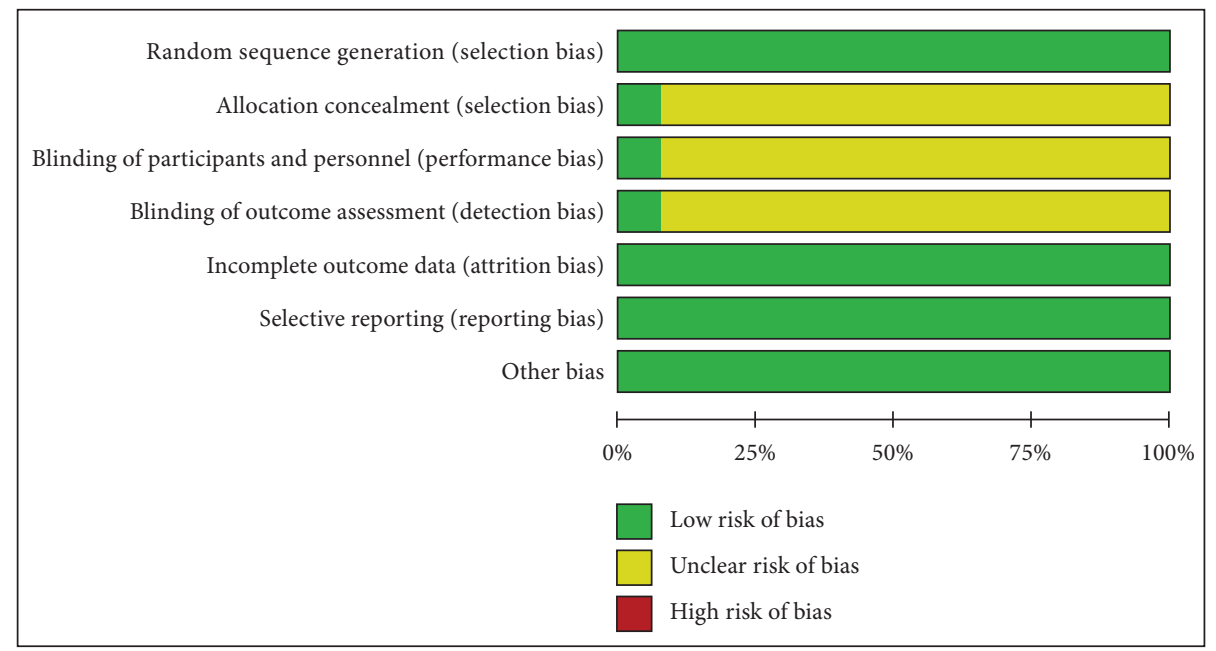

(a)

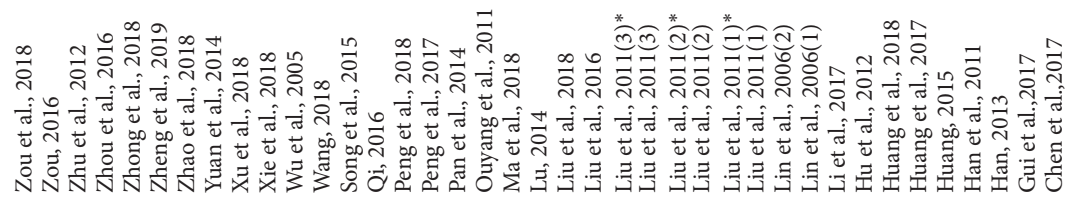

\begin{tabular}{|c|c|c|c|c|c|c|c|c|c|c|c|c|c|c|c|c|c|c|c|c|c|c|c|c|c|c|c|c|c|c|c|c|c|c|c|c|c|}
\hline$\oplus$ & $\oplus$ & $\oplus$ & $\oplus$ & $\oplus$ & $\oplus$ & $\oplus$ & $\oplus$ & $\oplus$ & $\oplus$ & $\oplus$ & $\oplus$ & $\oplus$ & $\oplus$ & $\oplus$ & (4) & $\oplus$ & $\oplus$ & $\oplus$ & $\oplus$ & $\oplus$ & $\oplus$ & $\oplus$ & $\oplus$ & $\oplus$ & $\oplus$ & $\oplus$ & $\oplus$ & $\oplus$ & & $\oplus$ & $\theta$ & $\oplus$ & $\oplus$ & $\oplus$ & $\oplus \mid$ & $\oplus$ & Random sequence generation (selection bias) \\
\hline$\sim$ & $\approx$ & $\oplus$ & $\approx$ & $\approx$ & (4) & $\approx$ & $\sim$ & (†) & $\sim$ & $\approx$ & $\approx$ & $\approx$ & $\approx$ & $\approx$ & $\sim$ & $\approx$ & $\approx$ & $\sim$ & $\approx$ & $\approx$ & $\approx$ & $\approx$ & $\sim 0$ & 2 & 20 & $\approx$ & 20 & 26 & 20 & $\approx \approx$ & $\approx$ & $\approx$ & $\sim$ & $\approx$ & 20 & $2 \approx$ & Allocation concealment (selection bias) \\
\hline 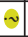 & 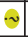 & $\theta$ & $\approx$ & $\approx$ & (†) & $\odot$ & 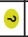 & (4) & $\approx$ & 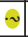 & $\odot$ & $\approx$ & $\approx$ & $\approx$ & $\approx$ & $\approx$ & $\approx$ & 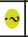 & 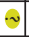 & $\approx$ & $\approx$ & $\approx$ & $\approx 6$ & 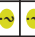 & $\approx$ & $\approx$ & 2 & $\approx$ & $\approx$ & $\sim \approx$ & 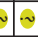 & $\approx$ & $\sim$ & $\approx$ & 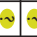 & $\sim \approx$ & Blinding of participants and personnel (performance bias) \\
\hline$\sim$ & $\sim$ & $\oplus$ & 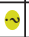 & $\sim$ & $\oplus$ & $\sim$ & 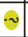 & $\oplus$ & ( & ( & 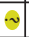 & $\sim$ & $\approx$ & $\odot$ & ( & $\approx$ & 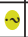 & $\approx$ & ( & $\sim$ & $\sim$ & $\odot$ & 26 & 20 & 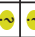 & $\sim$ & 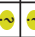 & 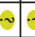 & $\sim 0$ & $\sim \approx$ & 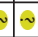 & $\approx$ & $\sim$ & $\sim$ & 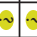 & $\approx \approx$ & Blinding of outcome assessment (detection bias) \\
\hline$\oplus$ & $\oplus$ & $\oplus$ & $\oplus$ & 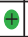 & $\oplus$ & $\oplus$ & (†) & (4) & $\oplus$ & $\oplus$ & $\oplus$ & $\oplus$ & $\oplus$ & $\oplus$ & (4) & $\oplus$ & $\oplus$ & $\oplus$ & $\oplus$ & $\oplus$ & (4) & $\oplus$ & $\theta$ & $\oplus$ & $\oplus$ & $\oplus$ & $\oplus$ & $\oplus$ & $\oplus$ & $\oplus \oplus$ & $\theta$ & $\oplus$ & $\oplus$ & $\oplus$ & 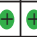 & $\oplus$ & Incomplete outcome data (attrition bias) \\
\hline$\oplus$ & $\oplus$ & $\oplus$ & $\oplus$ & $\oplus$ & $\oplus$ & $\oplus$ & $\oplus$ & $\oplus$ & $\oplus$ & $\oplus$ & $\theta$ & $\oplus$ & $\oplus$ & $\oplus$ & $\oplus$ & $\theta$ & $\oplus$ & $\oplus$ & $\oplus$ & $\oplus$ & $\oplus$ & $\theta$ & $\oplus$ & $\oplus \mid$ & $\oplus \mid$ & $\oplus \mid$ & $\oplus$ & $\oplus$ & +8 & $\oplus \oplus$ & $\theta$ & $\oplus$ & $\oplus$ & $\oplus$ & $\oplus$ & $\oplus$ & Selective reporting (reporting bias) \\
\hline$\oplus$ & $\oplus$ & $\theta$ & $\theta$ & $\oplus$ & $\oplus$ & $\oplus$ & $\theta$ & $\theta$ & $\oplus$ & $\oplus$ & $\oplus$ & $\oplus$ & (†) & $\oplus$ & (4) & (†) & $\theta$ & $\oplus$ & $\oplus$ & $\oplus$ & $\oplus$ & $\oplus$ & $\oplus$ & +6 & $\oplus$ & $\oplus$ & $\oplus$ & $\oplus$ & $\oplus$ & $\oplus$ & $\theta$ & $\oplus$ & $\oplus$ & $\oplus$ & 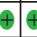 & $\oplus$ & Other bias \\
\hline
\end{tabular}

(b)

FIGURE 2: Risk of bias assessment. Notes: the experimental group of Liu 2011 (1) was divided into 2 groups, respectively: Liu 2011 (1) and Liu $2011(1)^{*}$; the experimental group of Liu 2011 (2) was divided into 2 groups, respectively: Liu 2011 (2) and Liu 2011 (2)*; the experimental group of Liu 2011 (3) was divided into 2 groups, respectively: Liu 2011 (3) and Liu 2011 (3)*.

among these studies was $\mathrm{chi}^{2}=3.07, P=0.55 ; I^{2}=0 \%$, $\mathrm{chi}^{2}=1.05, P=0.59 ; I^{2}=0 \%, \mathrm{chi}^{2}=1.16, P=0.56 ; I^{2}=0 \%$, and $\mathrm{chi}^{2}=3.02, P=0.55 ; I^{2}=0 \%$, respectively. Therefore, the fixed-effect model was applied to calculate the combined RR and $95 \%$ CI. After calculating, the combined RR and 95\% CI was $1.18(1.11,1.24) ; P<0.00001,1.36(1.21,1.53)$; $P<0.00001,1.34$ (1.15, 1.57); $P=0.0002$, and 1.21 (1.11, 1.32); $P<0.00001$, respectively, indicating a statistically significant difference between the experimental group and the control group. This result suggests that bushen huoxue, bushen zhuanggu, bushen jianpi, and buyi ganshen TCM herbal medicine combined with CWM in the treatment of POP can significantly improve clinical efficacy when compared with using CWM alone. The combined data showed that different specific therapies included in TCM herbal medicine combined with CWM were more effective than using CWM alone in improving effectiveness rates, with significant differences $(\mathrm{RR}=1.23 ; 95 \%$ CI $(1.18,1.29)$; $P<0.00001$ ) (Figure 3(b)).

3.3.3. Effectiveness Rates of Different Treatment Periods. Twenty-three literature studies reported effectiveness rates of different treatment periods $[21-23,26-28,30,31,33$,
34, 37-42, 44, 45, 47-50, 53]. Eleven literature studies reported treatment periods of less than 3 months [23, 27, $28,37,38,41,44,45,48-50]$. However, there was high statistical heterogeneity among studies $\left(\mathrm{chi}^{2}=22.88\right.$, $\left.P=0.01 ; I^{2}=56 \%\right)$. A sensitivity analysis was performed to identify the source of heterogeneity. By removing one trial [38], no heterogeneity was detected $\left(\mathrm{chi}^{2}=4.88, P=0.84\right.$; $\left.I^{2}=0 \%\right)$. We confirmed the accuracy of the data without publication bias after contacting the author. So, we adopted a random-effect model to calculate the combined RR and $95 \%$ CI as $1.35(1.27,1.43), P<0.00001$, indicating a statistically significant difference between the TS (TK) principle combined with the CWM group and the CWM alone group. It is suggested that using the TS (TK) principle combined with CWM for POP was better than using CWM alone in improving effectiveness rates when the treatment periods were less than 3 months. Twelve literature studies reported treatment periods of three to six months $[21,22,25,30,31,34,39,40,42,44,47,53]$. There was no statistical heterogeneity among studies $\left(\mathrm{chi}^{2}=7.92\right.$, $\left.P=0.85 ; I^{2}=0 \%\right)$. Therefore, the fixed-effect model was applied to calculate the combined RR and 95\% CI as 1.25 $(1.18,1.32), P<0.00001$, indicating a statistically significant 
Table 1: The search strategy used in the PubMed database.

\begin{tabular}{|c|c|}
\hline Serial number & Search items \\
\hline$\# 1$ & Bushen \\
\hline$\# 2$ & Tonifying Shen \\
\hline$\# 3$ & Bu Shen \\
\hline$\# 4$ & Yishen \\
\hline \#5 & Nourishing the kidney \\
\hline \#6 & Tonifying the kidney \\
\hline \#7 & Yi Shen \\
\hline$\# 8$ & Tonifying kidney \\
\hline \#9 & Nourishing kidney \\
\hline \#10 & Nourishing Shen \\
\hline$\# 11$ & Reinforcing the kidney \\
\hline$\# 12$ & Reinforcing kidney \\
\hline$\# 13$ & Reinforcing Shen \\
\hline \#14 & Invigorating the kidney \\
\hline \#15 & Invigorating kidney \\
\hline \#16 & Invigorating Shen \\
\hline$\# 17$ & Kidney-reinforcing \\
\hline \#18 & Kidney reinforcing \\
\hline \#19 & Shen reinforcing \\
\hline$\# 20$ & Shen-reinforcing \\
\hline$\# 21$ & Kidney-invigorating \\
\hline$\# 22$ & Kidney invigorating \\
\hline$\# 23$ & Shen-invigorating \\
\hline$\# 24$ & Kidney-tonifying \\
\hline$\# 25$ & Shen-tonifying \\
\hline \#26 & Kidney tonifying \\
\hline$\# 27$ & Shen tonifying \\
\hline$\# 28$ & Shen invigorating \\
\hline$\# 29$ & Invigorating Shen \\
\hline \#30 & $\# 1$ or $\# 2-\# 29$ \\
\hline \#31 & Primary osteoporosis \\
\hline \#32 & Osteoporosis \\
\hline \#33 & Age-related osteoporosis \\
\hline \#34 & Age-related osteoporosis \\
\hline \#35 & Osteoporosis, senile \\
\hline \#36 & Osteoporosis, involutional \\
\hline \#37 & Senile osteoporosis \\
\hline \#38 & Osteoporosis, age-related \\
\hline \#39 & Osteoporosis, age-related \\
\hline$\# 40$ & Bone loss, age-related \\
\hline \#41 & Age-related bone loss \\
\hline$\# 42$ & Age-related bone losses \\
\hline$\# 43$ & Bone loss, age-related \\
\hline$\# 44$ & Bone losses, age-related \\
\hline \#45 & $\# 31$ or $\# 32-\# 44$ \\
\hline \#46 & Randomized controlled trials \\
\hline \#47 & Randomized \\
\hline$\# 48$ & Randomly \\
\hline \#49 & Random \\
\hline \#50 & RCTs \\
\hline \#51 & $\# 46$ or \#47一\#50 \\
\hline \#52 & $\# 30$ and $\# 45$ and $\# 51$ \\
\hline
\end{tabular}

difference between the TS (TK) principle combined with the CWM group and the CWM alone group. It is suggested that using the TS (TK) principle combined with CWM for POP was better than using CWM alone in improving effectiveness rates when the treatment periods were three to six months. The pooled data showed that different treatment periods combined with CWM were more effective than using CWM alone in improving effectiveness rates, with significant differences $(\mathrm{RR}=1.29 ; 95 \%$ CI $(1.24,1.35) ; P<0.00001)$ (Figure 3(c)).

3.3.4. BMD (Lumbar Spine) of Different Treatment Periods. Twenty-one studies reported BMD (lumbar spine) of different treatment periods $[20,21,24,25,27,29,30,32,34$, $35,37-40,42,46,48-50,53,54]$. There were 8 studies which reported BMD (lumbar spine) of less than 3 months $[27,29,37,38,48-50,54]$, and 11 studies reported BMD (lumbar spine) of three to six months $[21,25,30,32,34,35,39,40,42,46,53]$. However, we detected high statistical heterogeneity among studies, $\mathrm{chi}^{2}=40.14, \quad P<0.00001 ; \quad I^{2}=83 \% \quad$ and $\quad \mathrm{chi}^{2}=69.18$, $P<0.00001 ; I^{2}=83 \%$. The source of heterogeneity may be related to different treatment methods. Therefore, we adopted the random-effect model, and meta-analysis showed that there was a significant difference between the experimental and control groups $(\mathrm{SMD}=0.83 ; 95 \% \mathrm{CI}(0.52$, $1.15) ; P<0.00001$ and $\mathrm{SMD}=0.56 ; 95 \%$ CI $(0.27,0.85)$; $P<0.00001)$. It is suggested that using the TS (TK) principle combined with CWM less than three months or three to six months can both improve the BMD of the lumbar spine and is better than using CWM alone. Two studies reported BMD (lumbar spine) of more than six months $[20,24]$, and there was high statistical heterogeneity among studies $\left(\mathrm{chi}^{2}=49.53, \quad P<0.00001 ; I^{2}=98 \%\right)$. We adopted the random-effect model, and meta-analysis showed that there was no significant difference between the experimental and control groups $(\mathrm{SMD}=1.36 ; 95 \% \mathrm{CI}(-1.33,4.0) ; P=0.32)$. In general, the meta-analysis showed that different treatment periods combined with CWM were more effective than using CWM alone in improving the BMD of the lumbar spine, with significant differences $(\mathrm{SMD}=0.71$; 95\% CI $(0.47,0.95) ; P<0.00001)$ (Figure 3(d)).

3.3.5. BMD (Proximal Femur (Femoral Neck or Total Hip)) of Different Treatment Periods. There were 15 studies which reported BMD (proximal femur (femoral neck or total hip)) of different treatment periods $[19,20,24,27,30,32,34,35,37,39,42,46,51-53]$. Two studies reported BMD (proximal femur (femoral neck or total hip)) of less than 3 months [27, 37]. High statistical heterogeneity among studies was detected $\left(\mathrm{chi}^{2}=10.41\right.$, $\left.P=0.001 ; I^{2}=90 \%\right)$; therefore, we adopted the randomeffect model, and meta-analysis showed that there was no significant difference between the experimental and control groups (SMD $=0.53 ; 95 \%$ CI $(-0.30,1.36) ; P=0.21)$. Ten studies reported BMD (proximal femur (femoral neck or total hip)) of three to six months $[30,32,34,35,39,42,46,51-53]$. There was high statistical heterogeneity among studies $\left(\mathrm{chi}^{2}=52.61, \quad P<0.00001\right.$; $\left.I^{2}=83 \%\right)$. We performed sensitivity analysis by removing one trial [35], and low heterogeneity was detected $\left(\right.$ chi $\left.^{2}=11.68, P=0.17 ; I^{2}=32 \%\right)$. The source of heterogeneity may be related to different treatment methods. So, a random-effect model was adopted. The results showed that using the TS (TK) principle combined with CWM three to 


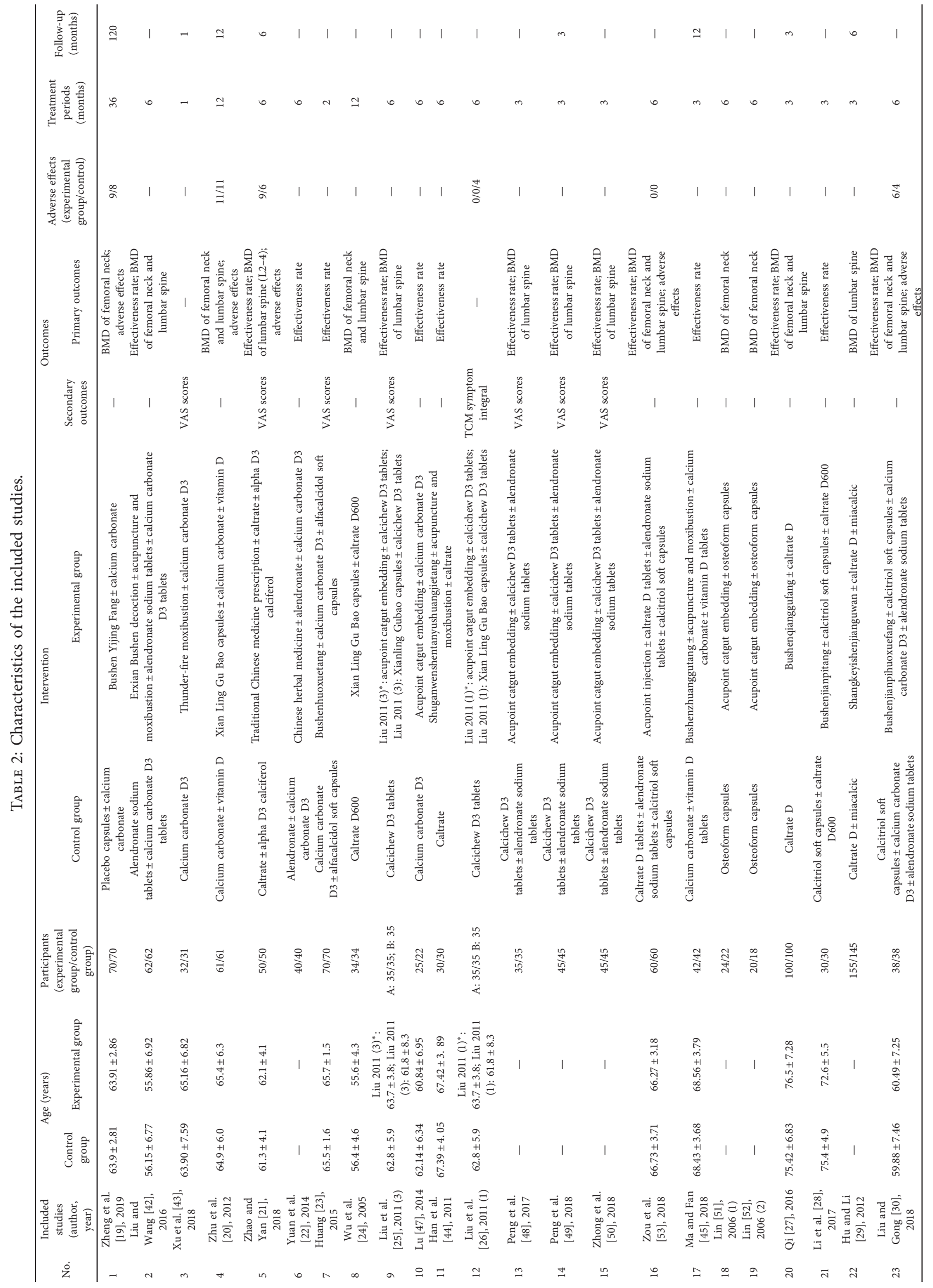




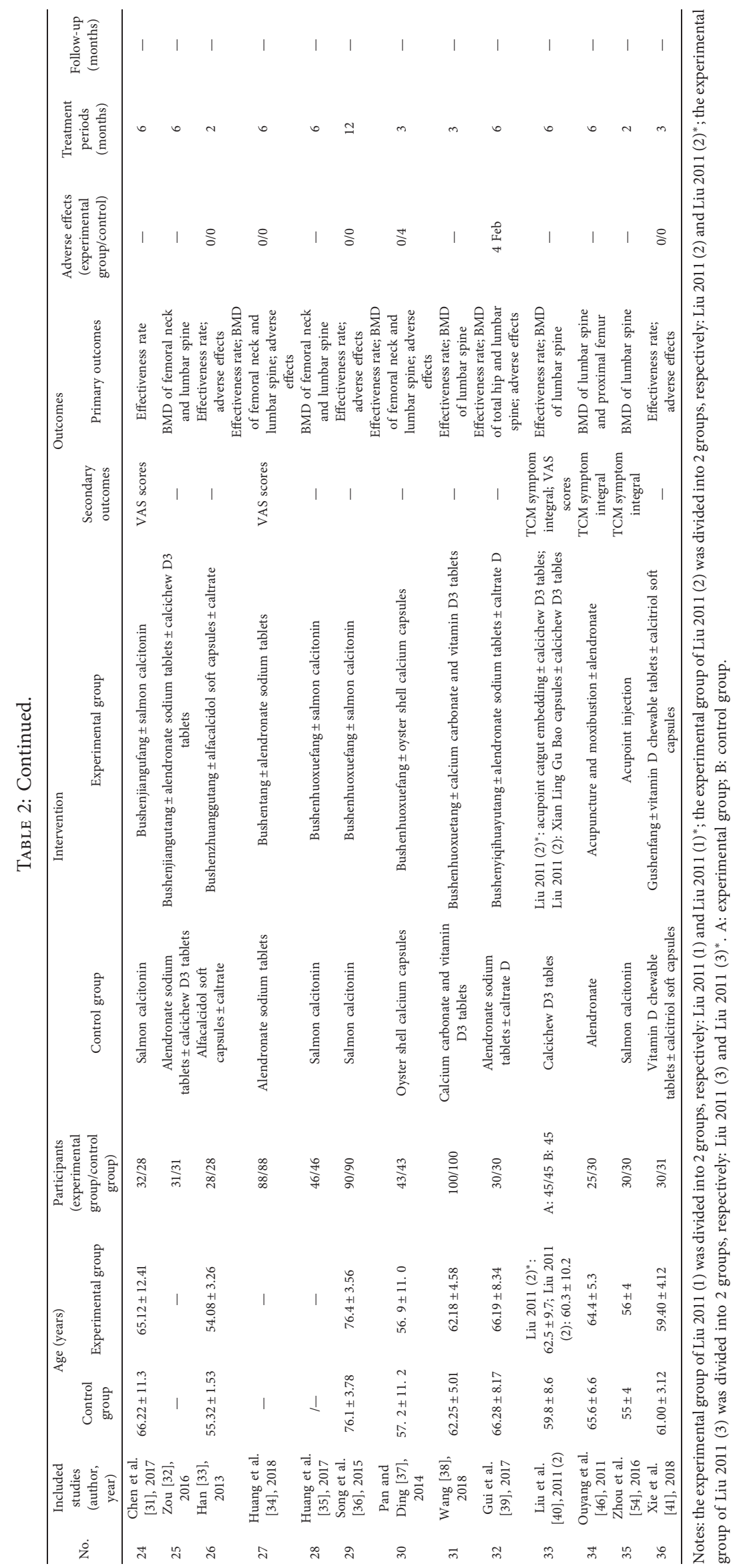




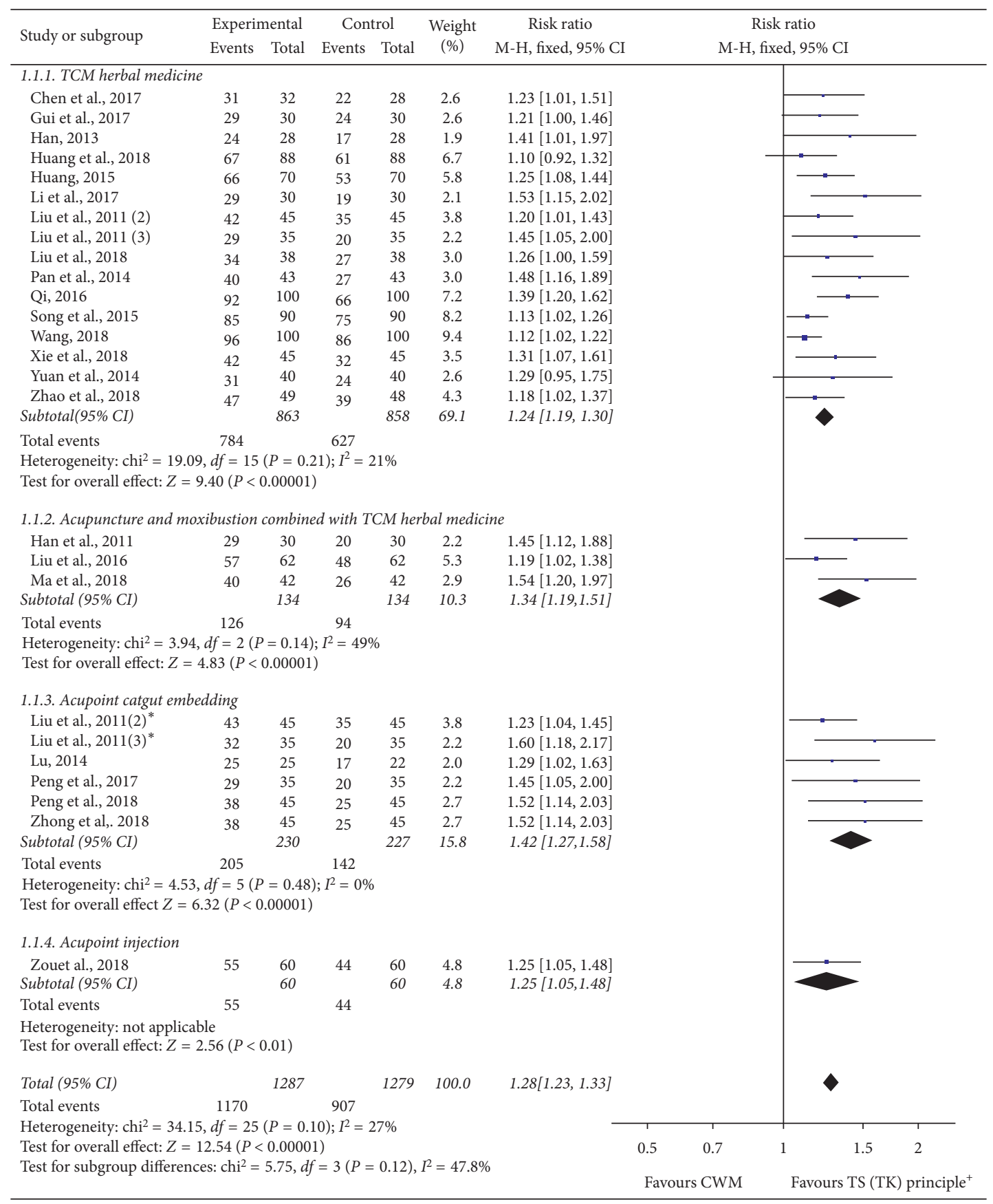

(a)

FIgURE 3: Continued. 


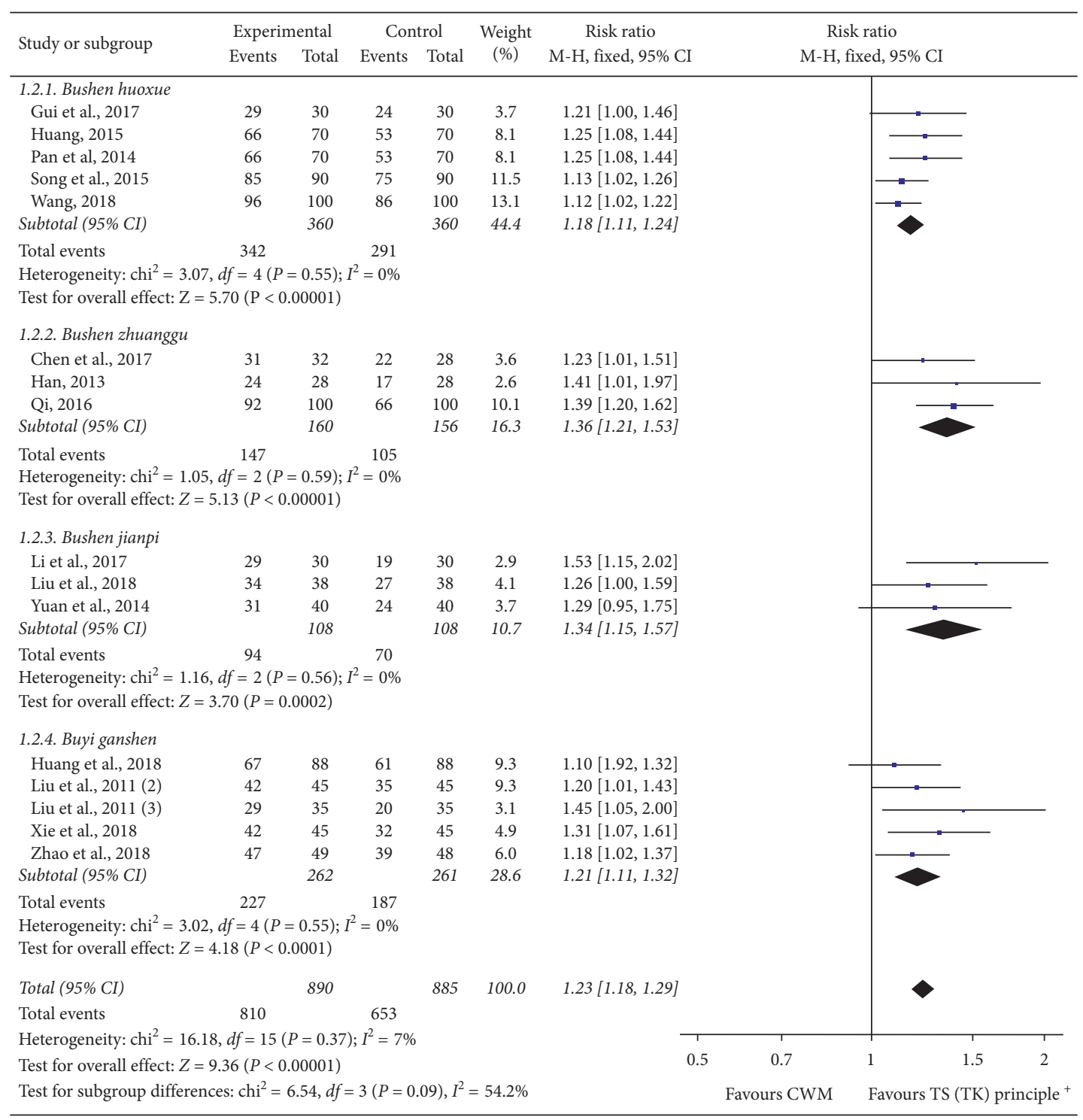

(b)

Figure 3: Continued. 


\begin{tabular}{|c|c|c|c|c|c|c|c|c|c|}
\hline \multirow{2}{*}{ Study or subgroup } & \multicolumn{2}{|c|}{ Experimental } & \multicolumn{2}{|c|}{ Control } & \multirow{2}{*}{$\begin{array}{l}\text { Weight } \\
(\%)\end{array}$} & \multirow{2}{*}{$\begin{array}{c}\text { Risk ratio } \\
\text { M-H, fixed, } 95 \% \text { CI }\end{array}$} & \multirow{2}{*}{\multicolumn{2}{|c|}{$\begin{array}{c}\text { Risk ratio } \\
\text { M-H, fixed, 95\% CI }\end{array}$}} & \\
\hline & Events & Total & Events & Total & & & & & \\
\hline \multicolumn{10}{|l|}{ 1.3.1. $\leq 3$ months } \\
\hline Han, 2013 & 24 & 28 & 17 & 28 & 2.0 & $1.41[1.01,1.97]$ & & & \\
\hline Huang, 2015 & 66 & 70 & 53 & 70 & 6.3 & $1.25[1.08,1.44]$ & & & \\
\hline Li et al., 2017 & 29 & 30 & 19 & 30 & 2.3 & $1.53[1.15,2.02]$ & & & \\
\hline Ma et al., 2018 & 40 & 42 & 26 & 42 & 3.1 & $1.54[1.20,1.97]$ & & & \\
\hline Pan et al., 2014 & 40 & 43 & 27 & 43 & 3.2 & $1.48[1.16,1.89]$ & & & \\
\hline Peng et al., 2017 & 29 & 35 & 20 & 35 & 2.4 & $1.45[1.05,2.00]$ & & & \\
\hline Peng et al., 2018 & 38 & 45 & 25 & 45 & 3.0 & $1.52[1.14,2.03]$ & & & \\
\hline Qi, 2016 & 92 & 100 & 66 & 100 & 7.9 & $1.39[1.20,1.62]$ & & & \\
\hline Wang, 2018 & 96 & 100 & 86 & 100 & 10.3 & $1.12[1.02,1.22]$ & & $\longrightarrow$ & \\
\hline Xie et al., 2018 & 42 & 45 & 32 & 45 & 3.8 & $1.31[1.07,1.61]$ & & & \\
\hline Zhong et al,. 2018 & 38 & 45 & 25 & 45 & 3.0 & $1.52[1.14,2.03]$ & & & \\
\hline Subtotal $(95 \%$ CI) & & 583 & & 583 & 47.4 & $1.35[1.27,1.43]$ & & & \\
\hline Total events & 534 & & 396 & & & & & & \\
\hline \multicolumn{10}{|c|}{ Heterogeneity: $\mathrm{chi}^{2}=22.88 d f=10(P=0.01) ; I^{2}=56 \%$} \\
\hline \multicolumn{10}{|c|}{ Test for overall effect: $Z=9.71(P<0.00001)$} \\
\hline \multicolumn{10}{|l|}{ 1.3.2. 3-6 months } \\
\hline Chen et al., 2017 & 31 & 32 & 22 & 28 & 2.8 & $1.23[1.01,1.51]$ & & & \\
\hline Gui et al., 2017 & 29 & 30 & 24 & 30 & 2.9 & $1.21[1.00,1.46]$ & & & \\
\hline Han et al., 2011 & 29 & 30 & 20 & 30 & 2.4 & $1.45[1.12,1.88]$ & & & \\
\hline Huang et al., 2018 & 67 & 88 & 61 & 88 & 7.3 & $1.10[0.92,1.32]$ & & & \\
\hline Liu et al., 2011 (2) & 42 & 45 & 35 & 45 & 4.2 & $1.20[1.01,1.43]$ & & & \\
\hline Liu et al., $2011(2)^{*}$ & 43 & 45 & 35 & 45 & 4.2 & $1.23[1.04,1.45]$ & & & \\
\hline Liu et al., 2011 (3) & 29 & 35 & 20 & 35 & 2.4 & $1.45[1.05,2.00]$ & & & \\
\hline Liu et al., $2011(3)^{*}$ & 32 & 35 & 20 & 35 & 2.4 & $1.60[1.18,2.17]$ & & & \\
\hline Liu et al., 2016 & 57 & 62 & 48 & 62 & 5.7 & $1.19[1.02,1.38]$ & & & \\
\hline Liu et al., 2018 & 34 & 38 & 27 & 38 & 3.2 & $1.26[1.00,1.59]$ & & & \\
\hline $\mathrm{Lu}, 2014$ & 25 & 25 & 17 & 22 & 2.2 & $1.29[1.02,1.63]$ & & & \\
\hline Yuan et al., 2014 & 31 & 40 & 24 & 40 & 2.9 & $1.29[0.95,1.75]$ & & & \\
\hline Zhao et al., 2018 & 47 & 49 & 39 & 48 & 4.7 & $1.18[1.02,1.37]$ & & & \\
\hline Zou et al., 2018 & 55 & 60 & 44 & 60 & 5.3 & $1.25[1.05,1.48]$ & & & \\
\hline Subtotal $(95 \%$ CI) & & 614 & & 606 & 52.6 & $1.25[1.18,1.32]$ & & & \\
\hline \multirow{2}{*}{\multicolumn{10}{|c|}{ Heterogeneity: chi $^{2}=7.92 d f=13(P=0.85) ; I^{2}=0 \%$}} \\
\hline & & & & & & & & & \\
\hline \multicolumn{10}{|c|}{ Test for overall effect: $Z=7.70(P<0.00001)$} \\
\hline Total (95\% CI) & & 1197 & & 1189 & 100.0 & $1.29[1.24,1.35]$ & & & \\
\hline Total events & 1085 & & 832 & & & & & & \\
\hline \multicolumn{7}{|c|}{ Heterogeneity: chi $^{2}=30.33 d f=24(P=0.17) ; I^{2}=21 \%$} & - & & \\
\hline \multicolumn{7}{|c|}{ Test for overall effect: $Z=12.32(P<0.00001)$} & 0.5 & 1 & 1.5 \\
\hline \multicolumn{7}{|c|}{ Test for subgroup differences: $\mathrm{chi}^{2}=3.57, d f=1(P=0.06), I^{2}=72.0 \%$} & Favours CWM & Favours Ts & S (TK) principle ${ }^{+}$ \\
\hline
\end{tabular}

(c)

FIgURE 3: Continued. 


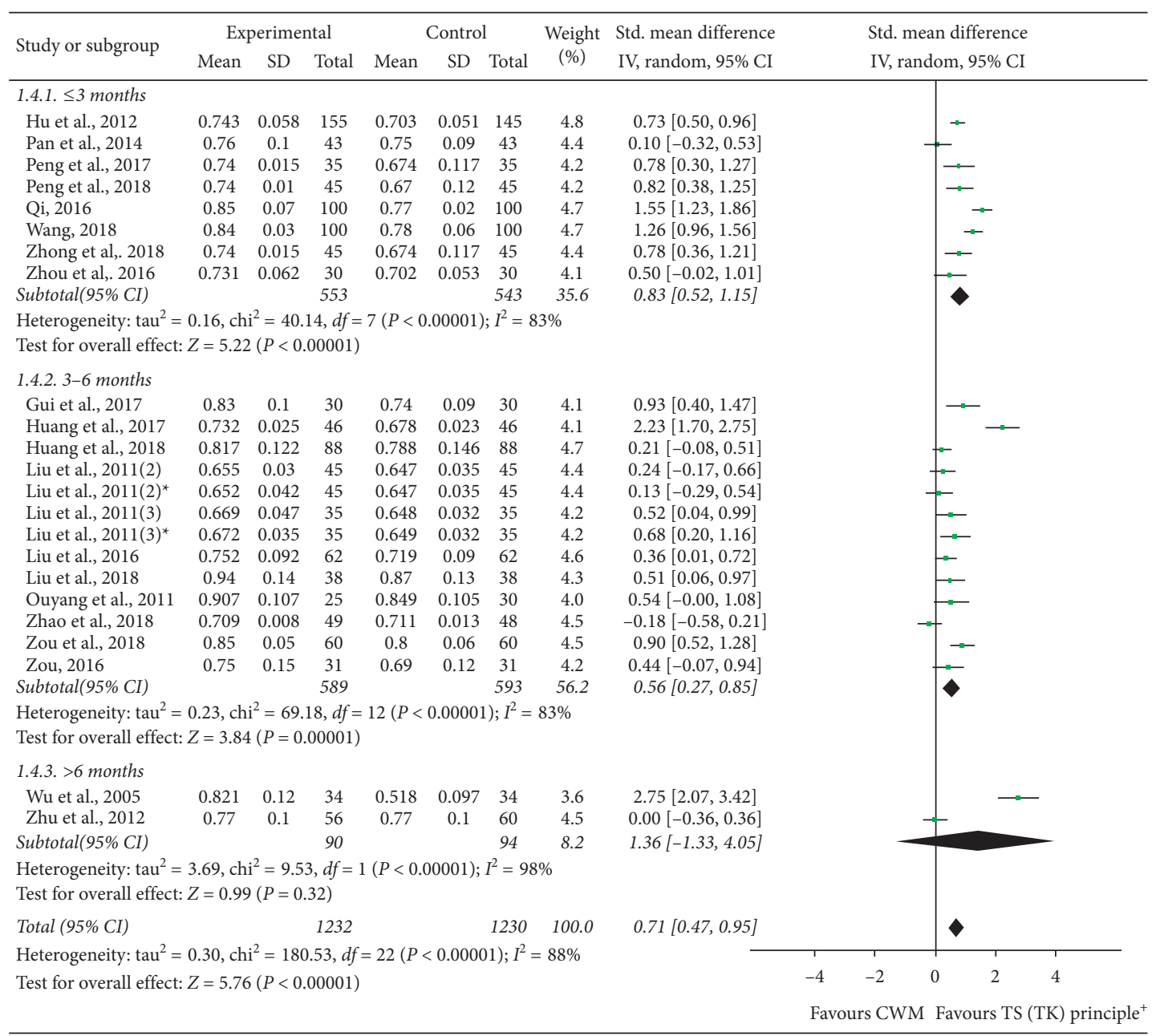

(d)

Figure 3: Continued. 


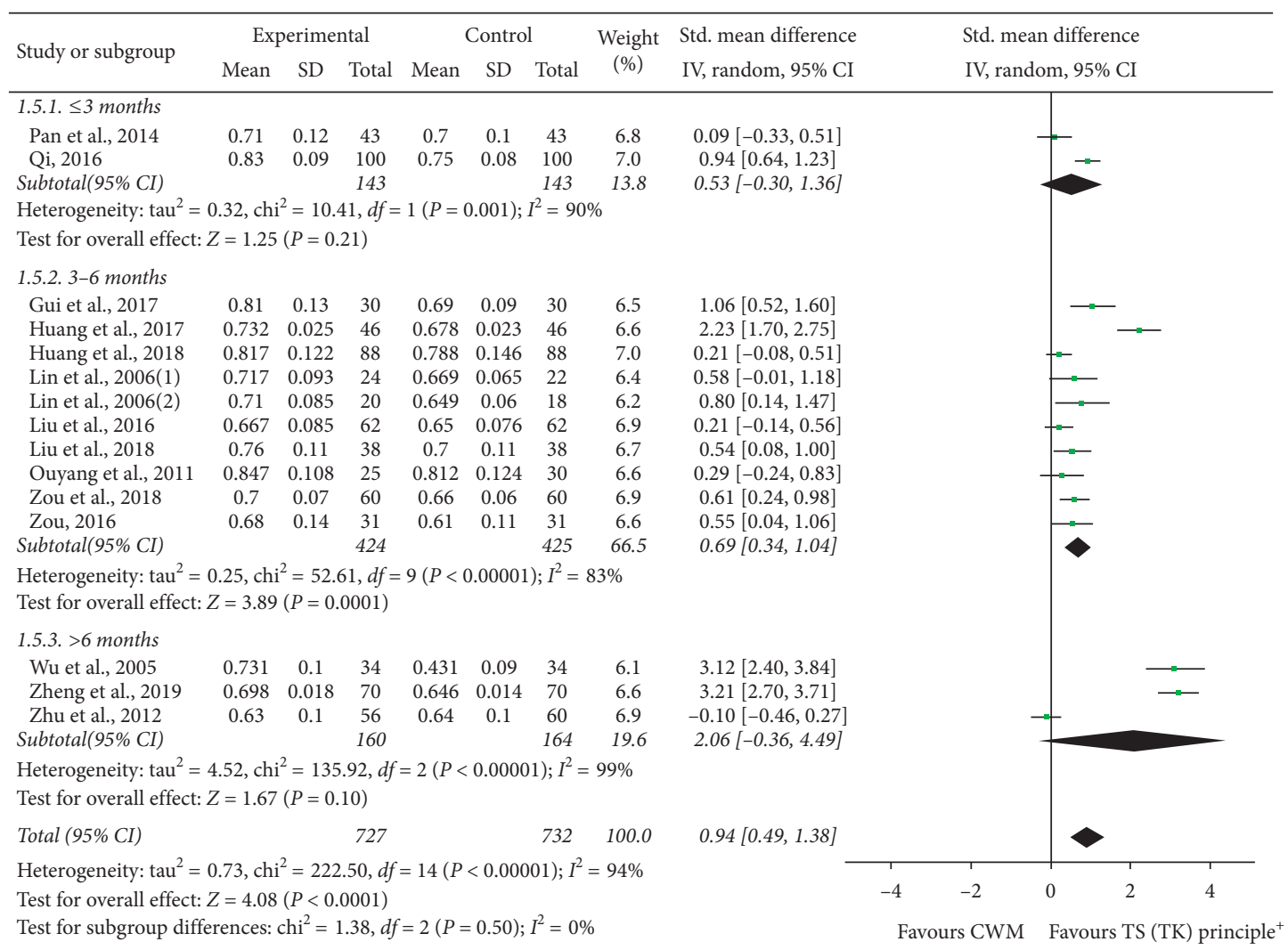

(e)

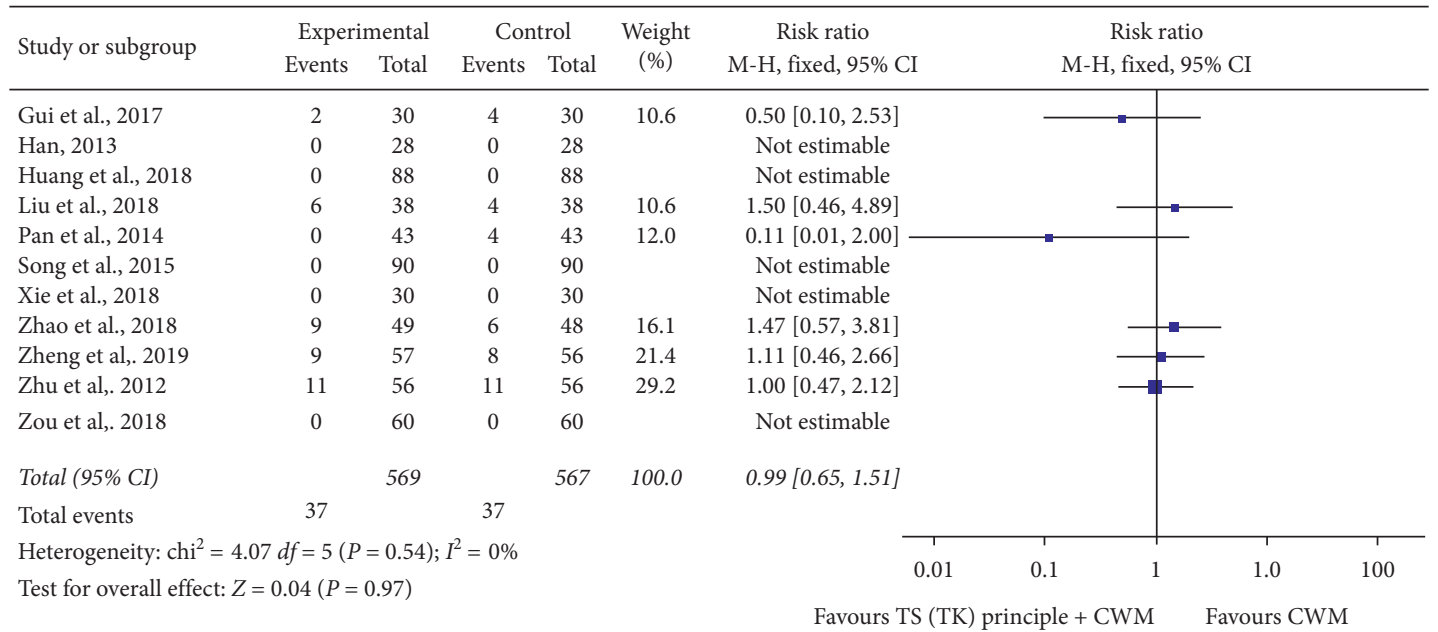

(f)

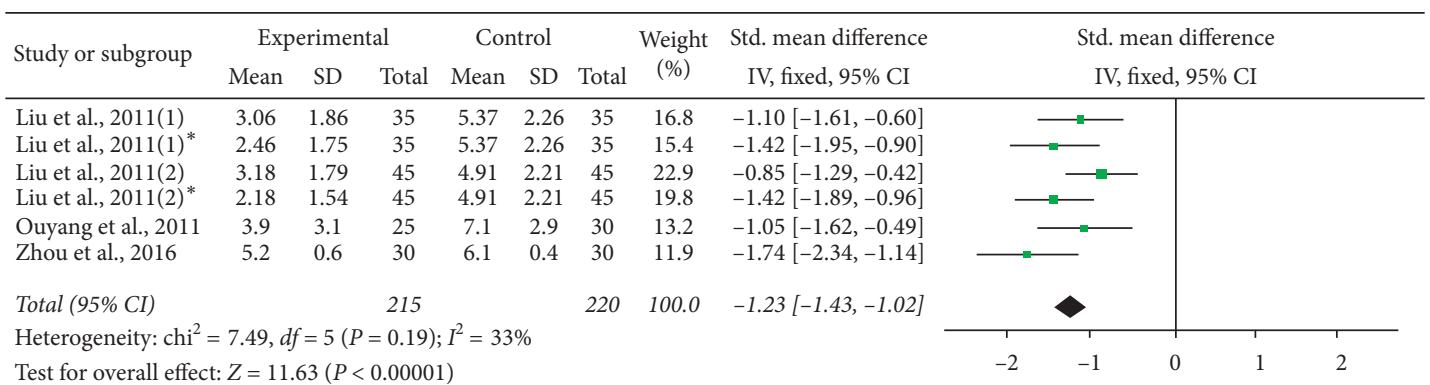

Favours TS (TK) principle + CWM Favours CWM

(g)

Figure 3: Continued. 


\begin{tabular}{|c|c|c|c|c|c|c|c|c|c|c|c|c|}
\hline \multirow{3}{*}{$\begin{array}{l}\text { Study or subgroup } \\
\text { Chen et al., } 2017\end{array}$} & \multicolumn{3}{|c|}{ Experimental } & \multicolumn{2}{|c|}{ Control } & \multirow{2}{*}{ Total } & \multirow{2}{*}{$\begin{array}{l}\text { Weight } \\
\text { (\%) }\end{array}$} & \multirow{2}{*}{$\begin{array}{l}\text { Std. mean difference } \\
\text { IV, random, 95\% CI }\end{array}$} & \multirow{2}{*}{\multicolumn{4}{|c|}{$\begin{array}{l}\text { Std. mean difference } \\
\text { IV, random, 95\% CI }\end{array}$}} \\
\hline & Mean & SD & Total & Mean & SD & & & & & & & \\
\hline & 2.14 & 1.42 & 32 & 3.65 & 1.47 & 28 & 8.6 & $-1.03[-1.57,-0.49]$ & & - & & \\
\hline Huang et al., 2018 & 1.73 & 0.25 & 88 & 2.32 & 0.18 & 88 & 8.6 & $-2.70[-3.11,-2.29]$ & & - & & \\
\hline Huang, 2015 & 1.15 & 0.03 & 70 & 2.14 & 0.16 & 70 & 8.3 & $-8.55[-9.62,-7.48]$ & . & & & \\
\hline Liu et al., 2011(2) & 3.34 & 0.71 & 45 & 2.02 & 0.66 & 45 & 8.6 & $1.91[1.41,2.41]$ & & & - & \\
\hline Liu et al., $2011(2)^{*}$ & 1.53 & 0.78 & 45 & 2.02 & 0.66 & 45 & 8.6 & $-0.67[-1.10,-0.25]$ & & - & & \\
\hline Liu et al., 2011(3) & 2.03 & 0.67 & 35 & 3.31 & 0.73 & 35 & 8.6 & $-1.81[-2.37,-1.25]$ & & - & & \\
\hline Liu et al., 2011(3)* & 1.5 & 0.82 & 35 & 3.31 & 0.73 & 35 & 8.5 & $-2.31[-2.92,-1.69]$ & & - & & \\
\hline Peng et al., 2017 & 2.238 & 0.164 & 35 & 4.165 & 0.202 & 35 & 7.6 & $-10.36[-12.19,-8.53]$ & & & & \\
\hline Peng et al., 2018 & 2.24 & 0.16 & 45 & 4.17 & 0.2 & 45 & 7.8 & $-10.57[-12.20,-8.93]$ & & & & \\
\hline Xu et al,. 2018 & 2.77 & 0.92 & 32 & 4 & 1.28 & 31 & 8.6 & $-1.09[-1.62,-0.56]$ & & - & & \\
\hline Zhao et al., 2018 & 1.4 & 0.5 & 49 & 1.9 & 0.4 & 48 & 8.6 & $-1.09[-1.52,-0.67]$ & & - & & \\
\hline Zhong et al,. 2018 & 2.238 & 0.164 & 45 & 4.166 & 0.202 & 45 & 7.8 & $-10.39[-12.00,-8.78]$ & & & & \\
\hline Total $(95 \%$ CI) & & & 556 & & & 550 & 100.0 & $-3.88[-5.29,-2.46]$ & & & & \\
\hline \multicolumn{9}{|c|}{ Heterogeneity: $\operatorname{tau}^{2}=6.01, \mathrm{chi}^{2}=733.80, d f=11(P<0.00001) ; I^{2}=99 \%$} & -10 & -5 & 5 & 10 \\
\hline
\end{tabular}

(h)

FIGURE 3: (a) Effectiveness rates of different treatment methods included in the TS (TK) principle. (b) Effectiveness rates of different specific therapies included in TCM herbal medicine. (c) Effectiveness rates of different treatment periods. (d) BMD (lumbar spine) of different treatment periods. (e) BMD (proximal femur (femoral neck or total hip)) of different treatment periods. (f) Adverse effects. (g) TCM symptom integral. (h) VAS scores. Notes: the experimental group of Liu 2011 (2) was divided into 2 groups, respectively: Liu 2011 (2) and Liu 2011 (2)*; the experimental group of Liu 2011 (3) was divided into 2 groups, respectively: Liu 2011 (3) and Liu 2011 (3)*

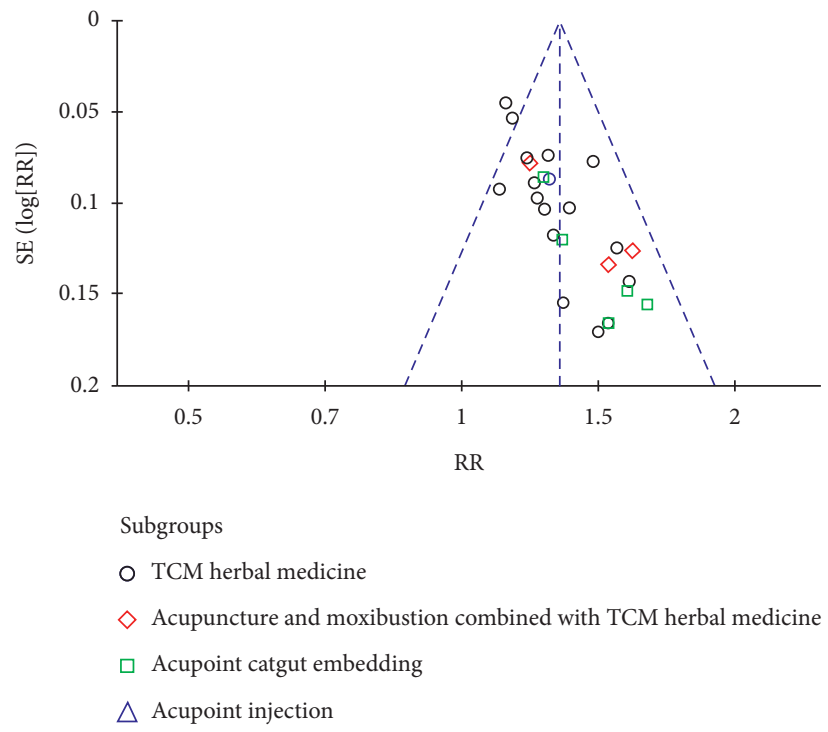

FIgURE 4: Funnel plot of effectiveness rates of different treatment methods included in the TS (TK) principle.

six months can improve the BMD of the proximal femur (femoral neck or total hip) and was better than using CWM alone $(\mathrm{SMD}=0.69 ; 95 \% \mathrm{CI}(0.34,1.04) ; P<0.00001)$. Three studies reported BMD of more than six months, and these showed that there was high heterogeneity $\left(\mathrm{chi}^{2}=135.92\right.$, $\left.P<0.00001 ; I^{2}=99 \%\right)[19,20,24]$. By removing one study [20], no heterogeneity was detected. The high heterogeneity may result from different treatment methods adopted by these studies. Thus, a random-effect model was adopted, and the results showed that there was no significant difference between the experimental and control groups $(\mathrm{SMD}=2.06$; 95\% CI $(0.36,4.49) ; P=0.10)$. The combined data showed that different treatment periods combined with CWM were more effective than using CWM alone in improving the $\mathrm{BMD}$ of the proximal femur (femoral neck or total hip), with significant differences $(\mathrm{SMD}=0.94 ; 95 \%$ CI $(0.49,1.38)$; $P<0.00001$ ) (Figure 3(e)).

3.3.6. Adverse Effects. Eleven studies reported adverse effects $[19-21,30,33,34,36,37,39,41,53]$, and there was no statistical heterogeneity among studies $\left(\mathrm{chi}^{2}=4.07\right.$, $\left.P=0.54 ; I^{2}=0 \%\right)$. Hence, the fixed-effect model was applied to calculate the combined RR and $95 \% \mathrm{CI}$ as $0.99(0.65,1.51)$, $P=0.97$, indicating no statistically significant difference between the experimental group and the control group. This result suggests that the TS (TK) principle combined with CWM or using CWM alone in the treatment of POP are both safe. Besides, the common adverse effects in the experimental group were gastrointestinal complaints, liver enzyme 


\begin{tabular}{|c|c|c|c|c|c|c|c|c|c|c|c|c|}
\hline \multicolumn{7}{|c|}{ Quality assessment } & \multicolumn{2}{|c|}{ No. of patients } & \multicolumn{2}{|r|}{ Effect } & \multirow[b]{2}{*}{ Quality } & \multirow[b]{2}{*}{ Importance } \\
\hline $\begin{array}{l}\text { No. of } \\
\text { studies }\end{array}$ & Design & $\begin{array}{c}\text { Risk of } \\
\text { bias }\end{array}$ & Inconsistecy & Indirectness & Imprecision & $\begin{array}{c}\text { Other } \\
\text { considerations }\end{array}$ & $\begin{array}{c}\text { TS (TK) principle } \\
\text { combined with } \\
\text { CWM }\end{array}$ & CWM & $\begin{array}{l}\text { Relative } \\
(95 \% \text { CI })\end{array}$ & Absolute & & \\
\hline \multicolumn{13}{|c|}{ Effectiveness rates of different treatment methods included in TS (TK) principle (follow-up 0-6 months) } \\
\hline \multirow[t]{2}{*}{25} & \multirow[t]{2}{*}{$\begin{array}{l}\text { randomised } \\
\text { trials }\end{array}$} & \multirow[t]{2}{*}{ serious $^{1}$} & \multirow[t]{2}{*}{$\begin{array}{c}\text { no serious } \\
\text { inconsistency }\end{array}$} & \multirow[t]{2}{*}{ serious $^{2}$} & \multirow[t]{2}{*}{$\begin{array}{l}\text { no serious } \\
\text { imprecision }\end{array}$} & \multirow[t]{2}{*}{$\begin{array}{c}\text { dose response } \\
\text { gradient }{ }^{3}\end{array}$} & $1170 / 1287(90.9 \%)$ & $\begin{array}{c}907 / 1279 \\
(70.9 \%)\end{array}$ & \multirow[t]{2}{*}{$\begin{array}{c}\text { RR } 1.28 \\
(1.23 \text { to } \\
1.33)\end{array}$} & $\begin{array}{c}199 \text { more per } 1000 \\
\text { (from } 163 \text { more to } \\
234 \text { more }\end{array}$ & \multirow[t]{2}{*}{ 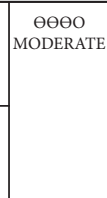 } & \multirow[t]{2}{*}{ CRITICAL } \\
\hline & & & & & & & & $70.2 \%$ & & $\begin{array}{c}197 \text { more per } 1000 \\
\text { (from } 161 \text { more to } \\
232 \text { more }\end{array}$ & & \\
\hline \multicolumn{13}{|c|}{ Effectiveness rates of different specific therapies included in TCM herbal medicine (follow-up 0-6 months) } \\
\hline \multirow[t]{2}{*}{16} & \multirow[t]{2}{*}{$\begin{array}{c}\text { randomised } \\
\text { trials }\end{array}$} & \multirow[t]{2}{*}{ serious $^{1}$} & \multirow[t]{2}{*}{$\begin{array}{c}\text { no serious } \\
\text { inconsistency }\end{array}$} & \multirow[t]{2}{*}{ serious $^{2}$} & \multirow[t]{2}{*}{$\begin{array}{l}\text { no serious } \\
\text { imprecision }\end{array}$} & \multirow[t]{2}{*}{$\begin{array}{c}\text { dose response } \\
\text { gradient }^{3}\end{array}$} & $810 / 890(91 \%)$ & $\begin{array}{l}653 / 885 \\
(73.8 \%)\end{array}$ & \multirow[t]{2}{*}{$\begin{array}{c}\text { RR } 1.23 \\
(1.18 \text { to } \\
1.29)\end{array}$} & $\begin{array}{c}170 \text { more per } 1000 \\
\text { (from } 133 \text { more to } \\
214 \text { more }\end{array}$ & \multirow[t]{2}{*}{$\begin{array}{c}\Theta \Theta \Theta O \\
\text { MODERATE }\end{array}$} & \multirow[t]{2}{*}{ CRITICAL } \\
\hline & & & & & & & & $73.4 \%$ & & $\begin{array}{c}169 \text { more per } 1000 \\
\text { (from } 132 \text { more to } \\
213 \text { more }\end{array}$ & & \\
\hline \multicolumn{13}{|c|}{ Effectiveness rates of different treatment periods (follow-up 0-6 months) } \\
\hline \multirow[t]{2}{*}{23} & \multirow[t]{2}{*}{$\begin{array}{c}\text { randomised } \\
\text { trials }\end{array}$} & \multirow[t]{2}{*}{ serious $^{1}$} & $\begin{array}{c}\text { no serious } \\
\text { inconsistency }\end{array}$ & serious $^{2}$ & $\begin{array}{l}\text { no serious } \\
\text { imprecision }\end{array}$ & $\begin{array}{c}\text { dose response } \\
\text { gradient }^{3}\end{array}$ & $1085 / 1197(90.6 \%)$ & $\begin{array}{c}832 / 1189 \\
(70 \%)\end{array}$ & $\begin{array}{l}\text { RR } 1.29 \\
(1.24 \text { to } \\
1.35)\end{array}$ & $\begin{array}{c}203 \text { more per } 1000 \\
\text { (from } 168 \text { more to } \\
245 \text { more }\end{array}$ & 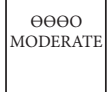 & CRITICAL \\
\hline & & & & & & & & $69.3 \%$ & & $\begin{array}{c}201 \text { more per } 1000 \\
\text { (from } 166 \text { more to } \\
243 \text { more }\end{array}$ & & \\
\hline & & & BMD (lur & bar spine) of d & fferent treatme & periods (follow- & p 0-120 months; bett & ter indicatec & by lower va & alues) & & \\
\hline 21 & $\begin{array}{c}\text { randomised } \\
\text { trials }\end{array}$ & serious $^{1}$ & $\begin{array}{c}\text { no serious } \\
\text { inconsistency }\end{array}$ & serious $^{2}$ & $\begin{array}{l}\text { no serious } \\
\text { imprecision }\end{array}$ & $\begin{array}{c}\text { strong } \\
\text { association }\end{array}$ & 1232 & 1230 & - & $\begin{array}{c}\text { SMD } 0.71 \text { higher } \\
(0.47 \text { to } 0.95 \text { higher })\end{array}$ & 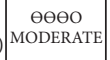 & CRITICAL \\
\hline & & BMD & ximal femur & noral neck o & total hip)) of d & erent treatment $\mathrm{p}$ & iods (follow-up 0-1 & months; b & etter indicat & ted by lower values) & & \\
\hline 15 & $\begin{array}{c}\text { randomised } \\
\text { trials }\end{array}$ & serious $^{1}$ & $\begin{array}{c}\text { no serious } \\
\text { inconsistency }\end{array}$ & serious $^{2}$ & $\begin{array}{l}\text { no serious } \\
\text { imprecision }\end{array}$ & $\begin{array}{c}\text { strong } \\
\text { association }\end{array}$ & 727 & 732 & - & $\begin{array}{c}\text { SMD } 0.94 \text { higher } \\
\text { (0.49 to } 1.38 \text { higher })\end{array}$ & 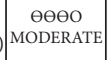 & CRITICAL \\
\hline & & & & & Advers & effects (follow up & $0-120$ months) & & & & & \\
\hline 11 & $\begin{array}{c}\text { randomised } \\
\text { trials }\end{array}$ & serious $^{1}$ & $\begin{array}{c}\text { no serious } \\
\text { inconsistency }\end{array}$ & serious $^{2}$ & $\begin{array}{l}\text { no serious } \\
\text { imprecision }\end{array}$ & none & $37 / 567(6.5 \%)$ & $\begin{array}{r}37 / 569 \\
(6.5 \%)\end{array}$ & $\begin{array}{l}\text { RR } 0.99 \\
(0.65 \text { to } \\
1.51)\end{array}$ & $\begin{array}{c}1 \text { fewer per } 1000 \\
\text { (from } 23 \text { fewer to } \\
33 \text { more) }\end{array}$ & $\begin{array}{c}\ominus \ominus 0 \mathrm{O} \\
\text { LOW }\end{array}$ & CRITICAL \\
\hline & & & & & & & & $9.3 \%$ & & $\begin{array}{c}1 \text { fewer per } 1000 \\
\text { (from } 33 \text { fewer to } \\
47 \text { more) }\end{array}$ & & \\
\hline & & & & & TCM sympton & ntegral (better in & licated by lower value & & & & & \\
\hline 4 & $\begin{array}{c}\text { randomised } \\
\text { trials }\end{array}$ & serious $^{1}$ & $\begin{array}{c}\text { no serious } \\
\text { inconsistency }\end{array}$ & serious $^{2}$ & $\begin{array}{l}\text { no serious } \\
\text { imprecision }\end{array}$ & $\begin{array}{c}\text { strong } \\
\text { association }\end{array}$ & 215 & 220 & - & $\begin{array}{c}\text { SMD } 1.23 \text { lower } \\
(1.43 \text { to } 1.02 \text { lower })\end{array}$ & 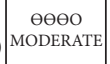 & IMPORTANT \\
\hline & & & & VAs & scores (follow & $0-6$ months; bet & er indicated by lower & values) & & & & \\
\hline 10 & $\begin{array}{c}\text { randomised } \\
\text { trials }\end{array}$ & serious $^{1}$ & $\begin{array}{c}\text { no serious } \\
\text { inconsistency }\end{array}$ & serious $^{2}$ & $\begin{array}{l}\text { no serious } \\
\text { imprecision }\end{array}$ & $\begin{array}{c}\text { strong } \\
\text { association }\end{array}$ & 556 & 550 & - & $\begin{array}{l}\text { SMD } 3.88 \text { lower } \\
\text { (5.29 to } 2.46 \text { lower) }\end{array}$ & MODERATE & IMPORTANT \\
\hline
\end{tabular}

1: Most of the literature can't be double blinded

2 : Surrogates measures

3: Increasing the intensity of intervention would increase the efficacy

$4: \mathrm{SMD}>0.70$ represents a large effect

FIgURE 5: GRADE evidence profile.

abnormal, hypertension, joint pain, stomach discomfort, nausea, vomiting, headache, musculoskeletal pain, etc. The adverse effects in the control group included gastrointestinal complaints, liver enzyme abnormal, hypertension, nausea, and vomiting (Figure 3(f)).
3.3.7. TCM Symptom Integral. The TCM symptom integral was established according to the Clinical Research Guidance of New Chinese Herbal Medicine [55]. Four studies reported TCM symptom integral $[26,40,46,54]$, and there was low statistical heterogeneity among studies $\left(\mathrm{chi}^{2}=7.49\right.$, 
TABLE 3: Brief table of risk assessment.

\begin{tabular}{|c|c|c|c|c|c|c|c|}
\hline \multirow[b]{2}{*}{ No. } & \multicolumn{7}{|c|}{ Risk of bias assessment (yes/no/unclear) } \\
\hline & $\begin{array}{c}\text { Included } \\
\text { studies (first } \\
\text { author, year) } \\
\end{array}$ & $\begin{array}{l}\text { Random sequence } \\
\text { generation }\end{array}$ & $\begin{array}{c}\text { Allocation } \\
\text { concealment }\end{array}$ & $\begin{array}{c}\text { Blinding of participants, } \\
\text { personnel, and outcome } \\
\text { assessors }\end{array}$ & $\begin{array}{l}\text { Incomplete } \\
\text { outcome data }\end{array}$ & $\begin{array}{l}\text { Selective } \\
\text { outcome } \\
\text { reporting }\end{array}$ & $\begin{array}{c}\text { Other } \\
\text { sources of } \\
\text { bias }\end{array}$ \\
\hline 1 & $\begin{array}{l}\text { Zheng et al. } \\
\text { [19], } 2019\end{array}$ & $\begin{array}{l}\text { Randomization } \\
\text { number table }\end{array}$ & Yes & Yes & Yes & No & No \\
\hline 2 & $\begin{array}{l}\text { Liu and Wang } \\
\text { [42], } 2016\end{array}$ & $\begin{array}{l}\text { Randomization } \\
\text { number table }\end{array}$ & Unclear & Unclear & Yes & No & No \\
\hline 3 & $\begin{array}{l}\text { Xu et al. [43], } \\
2018\end{array}$ & $\begin{array}{c}\text { Draw opaque envelope } \\
\text { randomly }\end{array}$ & Yes & Yes & Yes & No & No \\
\hline 4 & $\begin{array}{l}\text { Zhu et al. [20], } \\
2012\end{array}$ & $\begin{array}{l}\text { Using a computer } \\
\text { random number } \\
\text { generator }\end{array}$ & Yes & Yes & Yes & No & No \\
\hline 5 & $\begin{array}{l}\text { Zhao et al. [21], } \\
2018\end{array}$ & $\begin{array}{l}\text { Randomization } \\
\text { number table }\end{array}$ & Unclear & Unclear & Yes & No & No \\
\hline 6 & $\begin{array}{c}\text { Yuan et al. [22], } \\
2014\end{array}$ & $\begin{array}{l}\text { Randomization } \\
\text { number table }\end{array}$ & Unclear & Unclear & Yes & No & No \\
\hline 7 & $\begin{array}{l}\text { Huang [23] } \\
2015\end{array}$ & $\begin{array}{l}\text { Randomization } \\
\text { number table }\end{array}$ & Unclear & Unclear & Yes & No & No \\
\hline 8 & $\begin{array}{l}\text { Wu et al. [24], } \\
2005\end{array}$ & $\begin{array}{l}\text { Randomization } \\
\text { number table }\end{array}$ & Unclear & Unclear & Yes & No & No \\
\hline 9 & $\begin{array}{l}\text { Liu et al. [25], } \\
2011 \text { (3) }\end{array}$ & $\begin{array}{l}\text { Randomization } \\
\text { number table }\end{array}$ & Unclear & Unclear & Yes & No & No \\
\hline 10 & Lu [47], 2014 & $\begin{array}{l}\text { Doll's clinical case } \\
\text { random table }\end{array}$ & Unclear & Unclear & Yes & No & No \\
\hline 11 & $\begin{array}{c}\text { Han et al. [44], } \\
2011\end{array}$ & $\begin{array}{c}\text { Randomization } \\
\text { number table }\end{array}$ & Unclear & Unclear & Yes & No & No \\
\hline 12 & $\begin{array}{c}\text { Liu et al. [26], } \\
2011 \text { (1) }\end{array}$ & $\begin{array}{l}\text { Randomization } \\
\text { number table }\end{array}$ & Unclear & Unclear & Yes & No & No \\
\hline 13 & $\begin{array}{c}\text { Peng et al. [48], } \\
2017\end{array}$ & $\begin{array}{c}\text { Randomization } \\
\text { number table }\end{array}$ & Unclear & Unclear & Yes & No & No \\
\hline 14 & $\begin{array}{l}\text { Peng et al. [49], } \\
2018\end{array}$ & $\begin{array}{l}\text { Randomization } \\
\text { number table }\end{array}$ & Unclear & Unclear & Yes & No & No \\
\hline 15 & $\begin{array}{l}\text { Zhong et al. } \\
\text { [50], } 2018\end{array}$ & $\begin{array}{l}\text { Randomization } \\
\text { number table }\end{array}$ & Unclear & Unclear & Yes & No & No \\
\hline 16 & $\begin{array}{l}\text { Zou et al. [53], } \\
2018\end{array}$ & $\begin{array}{l}\text { Randomization } \\
\text { number table }\end{array}$ & Unclear & Unclear & Yes & No & No \\
\hline 17 & $\begin{array}{l}\text { Ma et al. [45], } \\
\qquad 2018\end{array}$ & Drawing of lots & Unclear & Unclear & Yes & No & No \\
\hline 18 & $\begin{array}{c}\text { Lin [51], } 2006 \\
(1)\end{array}$ & $\begin{array}{l}\text { Randomization } \\
\text { number table }\end{array}$ & Unclear & Unclear & Yes & No & No \\
\hline 19 & $\begin{array}{c}\text { Lin [52], } 2006 \\
(2)\end{array}$ & $\begin{array}{l}\text { Randomization } \\
\text { number table }\end{array}$ & Unclear & Unclear & Yes & No & No \\
\hline 20 & Qi [27], 2016 & $\begin{array}{l}\text { Randomization } \\
\text { number table }\end{array}$ & Unclear & Unclear & Yes & No & No \\
\hline 21 & $\begin{array}{l}\text { Li et al. [28], } \\
2017\end{array}$ & $\begin{array}{l}\text { Randomization } \\
\text { number table }\end{array}$ & Unclear & Unclear & Yes & No & No \\
\hline 22 & $\begin{array}{l}\mathrm{Hu} \text { and } \mathrm{Li}[29] \\
2012\end{array}$ & $\begin{array}{l}\text { Randomization } \\
\text { number table }\end{array}$ & Unclear & Unclear & Yes & No & No \\
\hline 23 & $\begin{array}{l}\text { Liu and Gong } \\
\text { [30], } 2018\end{array}$ & $\begin{array}{l}\text { Randomization } \\
\text { number table }\end{array}$ & Unclear & Unclear & Yes & No & No \\
\hline 24 & $\begin{array}{l}\text { Chen et al. [31], } \\
2017\end{array}$ & Coin tossing & Unclear & Unclear & Yes & No & No \\
\hline 25 & Zou [32], 2016 & $\begin{array}{l}\text { Randomization } \\
\text { number table }\end{array}$ & Unclear & Unclear & Yes & No & No \\
\hline 26 & Han [33], 2013 & Coin tossing & Unclear & Unclear & Yes & No & No \\
\hline 27 & $\begin{array}{l}\text { Huang et al. } \\
{[34], 2018}\end{array}$ & $\begin{array}{l}\text { Randomization } \\
\text { number table }\end{array}$ & Unclear & Unclear & Yes & No & No \\
\hline 28 & $\begin{array}{l}\text { Huang et al. } \\
{[35], 2017}\end{array}$ & $\begin{array}{l}\text { Randomization } \\
\text { number table }\end{array}$ & Unclear & Unclear & Yes & No & No \\
\hline
\end{tabular}


TABle 3: Continued.

\begin{tabular}{|c|c|c|c|c|c|c|c|}
\hline \multicolumn{8}{|c|}{ Risk of bias assessment (yes/no/unclear) } \\
\hline No. & $\begin{array}{c}\text { Included } \\
\text { studies (first } \\
\text { author, year) }\end{array}$ & $\begin{array}{l}\text { Random sequence } \\
\text { generation }\end{array}$ & $\begin{array}{c}\text { Allocation } \\
\text { concealment }\end{array}$ & $\begin{array}{l}\text { Blinding of participants, } \\
\text { personnel, and outcome } \\
\text { assessors }\end{array}$ & $\begin{array}{c}\text { Incomplete } \\
\text { outcome data }\end{array}$ & $\begin{array}{l}\text { Selective } \\
\text { outcome } \\
\text { reporting }\end{array}$ & $\begin{array}{c}\text { Other } \\
\text { sources of } \\
\text { bias }\end{array}$ \\
\hline 29 & $\begin{array}{l}\text { Song et al. [36], } \\
2015\end{array}$ & Draw lots randomly & Unclear & Unclear & Yes & No & No \\
\hline 30 & $\begin{array}{l}\text { Pan and Ding } \\
\text { [37], } 2014\end{array}$ & $\begin{array}{l}\text { Randomization } \\
\text { number table }\end{array}$ & Unclear & Unclear & Yes & No & No \\
\hline 31 & Wang [38], 2018 & $\begin{array}{c}\text { Randomization } \\
\text { number table }\end{array}$ & Unclear & Unclear & Yes & No & No \\
\hline 32 & $\begin{array}{l}\text { Gui et al. [39], } \\
\qquad 2017\end{array}$ & $\begin{array}{l}\text { Randomization } \\
\text { number table }\end{array}$ & Unclear & Unclear & Yes & No & No \\
\hline 33 & $\begin{array}{l}\text { Liu et al. [40], } \\
2011 \text { (2) }\end{array}$ & $\begin{array}{c}\text { Randomization } \\
\text { number table }\end{array}$ & Unclear & Unclear & Yes & No & No \\
\hline 34 & $\begin{array}{c}\text { Ouyang et al. } \\
{[46], 2011}\end{array}$ & $\begin{array}{l}\text { Randomization } \\
\text { number table }\end{array}$ & Unclear & Unclear & Yes & No & No \\
\hline 35 & $\begin{array}{l}\text { Zhou et al. [54], } \\
2016\end{array}$ & $\begin{array}{l}\text { Randomization } \\
\text { number table }\end{array}$ & Unclear & Unclear & Yes & No & No \\
\hline 36 & $\begin{array}{l}\text { Xie et al. [41], } \\
2018\end{array}$ & $\begin{array}{l}\text { Randomization } \\
\text { number table }\end{array}$ & Unclear & Unclear & Yes & No & No \\
\hline
\end{tabular}

$\left.P=0.19 ; I^{2}=33 \%\right)$. Therefore, the fixed-effect model was applied. The meta-analysis showed that there was a statistically significant difference between the experimental group and the control group (SMD $=-1.23$; 95\% CI $(-1.43,-1.02)$; $P<0.00001)$. This result suggests that the TS (TK) principle combined with CWM in the treatment of POP can significantly improve TCM symptom integral when compared with using CWM alone (Figure $3(\mathrm{~g})$ ).

3.3.8. VAS Scores. There were 10 studies which reported VAS scores [21, 23, 25, 31, 34, 40, 43, 48-50]. The result showed that there was high statistical heterogeneity among studies $\left(\mathrm{chi}^{2}=733.80, P<0.00001 ; I^{2}=99 \%\right)$, so we adopted a random-effect model. The combined data showed that there was a statistically significant difference between the experimental group and the control group $(\mathrm{SMD}=-3.88 ; 95 \%$ CI $(-5.29,-2.46) ; P<0.00001)$. This result suggests that the TS (TK) principle combined with CWM in the treatment of POP was more effective than using CWM alone in improving VAS scores (Figure 3(h)).

3.3.9. Publication Bias. The funnel plots were generated for studies with data on the effectiveness rates of different treatment methods included in the TS (TK) principle. The results showed that most of the points in the funnel plots were symmetrical. However, two points were outside the 95\% CIs, which indicates that there may have been publication bias in our studies and that might influence the results of our analysis, as can be seen in Figure 4 .

3.3.10. Quality of Evidence. There were 8 results for levels of evidence in our study. The detailed GRADE evidence profile of results is shown in Figure 5.

\section{Discussion}

4.1. Summary of Main Results. There were 36 included RCTs with 3617 participants in our research. Even though most of the trials had small sample sizes and poor methodological quality, our meta-analysis reached the following results: (1) analysis of the pooled data showed a consistently superior effect of the TS (TK) principle combined with CWM in terms of total effectiveness rates, BMD of the lumbar spine and proximal femur (femoral neck or total hip), TCM symptom integral, and VAS scores when compared to using CWM alone; (2) in terms of adverse effects, the same safety was obtained for the TS (TK) principle combined with CWM or using CWM alone for POP; (3) different treatment methods included in the TS (TK) principle combined with CWM were more effective than using CWM alone in improving effectiveness rates. It should be noted that since the sample size of acupoint injection effectiveness rates was small, the combined RR and 95\% CI was reported from the original study, not from the meta-analysis results; (4) in respect of TCM herbal medicine, different specific therapies combined with CWM for POP were more effective than using CWM alone; (5) compared with using CWM alone, the TS (TK) principle combined with CWM was more effective for POP in the aspect of different treatment periods; (6) in the three-to six-month treatment period, the TS (TK) principle combined with CWM for POP in terms of BMD of the lumbar spine and proximal femur (femoral neck or total hip) was better than using CWM alone; (7) according to the guideline of GRADE, the effectiveness rates of different treatment methods included in the TS (TK) principle, effectiveness rates of different specific therapies included in TCM herbal medicine, effectiveness rates of different treatment periods, BMD (lumbar spine) of different treatment periods, BMD (proximal femur (femoral neck or total hip)) of different treatment periods, TCM 
symptom integral, and VAS scores were moderate level of evidence. The adverse effects were low of evidence.

4.2. Analysis of TS (TK) Principle. TCM has been used in a range of medical management and health interventions in China and any other Asian countries for over 2500 years. POP patients are usually seeking TCM treatment, when the therapeutic effect of CWM is unsatisfactory. According to TCM theory, the establishment of a therapeutic principle is based on TCM syndromes, not symptoms. The clinical diagnosis of the TCM syndrome relies on the gathering of clinical information through inspection, auscultation and olfaction, inquiry, and palpation [56]. For POP, the fundamental physiopathological changes of the bone depend on whether Shen (kidney) essence is sufficient or not. Therefore, the TS (TK) principle is the key point to prevent and treat Shen (kidney)-deficiency syndrome of POP [57-60]. On the one hand, the effective mechanisms of pharmaceutical treatment included in the TS (TK) principle on POP have been demonstrated in the voluminous literature. Icariin (ICA), similar to estrogen, has a definite antiosteoporotic effect [61, 62]. Besides, oleanolic acid (OA) and psoralen have been reported to prevent bone loss by inhibiting osteoclast formation [63-65]. On the other hand, in respect of nonpharmaceutical treatment included in the TS (TK) principle, experiments have demonstrated that acupuncture could alleviate osteoporosis by regulating the expression of members in OPG/RANKL, Wnt/ $\beta$-catenin, and MAPK pathways [66]. Acupoint catgut embedding could regulate the hypothalamic-pituitary-ovarian axis to raise the serum $E_{2}$ level which would be significant in preventing osteoporosis [67]. Even experiments have shown that acupoint catgut embedding ameliorated the ovariectomization- (OVX-) caused metabonomic changes more effectively than hormone replacement therapy (HRT) with nilestriol [68]. Also, nonpharmaceutical treatment could alleviate related symptoms of osteoporosis and improve the quality of life [43]. Therefore, the TS (TK) principle would be a promising approach for POP, and it can not only improve physiological and biochemical indicators but also alleviate the TCM syndromes [16].

4.3. Limitations of Research. However, some limitations in our meta-analysis should be mentioned. (1) Although all of our included studies were RCTs, the methodological quality of them was generally improvable. Most of them failed to describe the blinding methods in detail, allocation, and concealment methods. (2) Among 36 studies, only 8 studies reported follow-up. The longest follow-up period was 120 months, and no further follow-up data were collected. The long-term effect of the TS (TK) principle for POP should be further studied. (3) For POP, fracture incidence should be the most patient-important outcomes. However, no fracture incidence data were collected in our studies. It is necessary to focus on fracture incidence as a patientimportant outcome in further studies. (4) The studies included in this analysis were insufficient, especially in terms of subgroup analysis. Thus, potential publication bias probably exists. (5) The high heterogeneity among studies may be related to the different treatment methods, treatment periods, and even the skill level of the practitioners. It is a common problem in the research of TCM therapy. (6) The study for different specific therapies of TCM herbal medicine was insufficient, and further data mining should be carried out.

\section{Conclusion}

In summary, our meta-analysis suggests that using the TS (TK) principle combined with CWM for POP is effective and safe. However, the limitation in the quality and quantity of the included RCTs might weaken the overall reliability of this conclusion. Therefore, large-volume, well-designed RCTs with extensive follow-up are awaited to confirm and update the findings of this analysis.

\section{Abbreviations}

$\begin{array}{ll}\text { POP: } & \text { Primary osteoporosis } \\ \text { OP: } & \text { Osteoporosis } \\ \text { PMOP: } & \text { Postmenopausal osteoporosis } \\ \text { CWM: } & \text { Conventional Western medicines } \\ \text { TCM: } & \text { Traditional Chinese Medicine } \\ \text { TS (TK) } & \text { Tonifying-Shen (kidney) principle } \\ \text { principle: } & \\ \text { RCTs: } & \text { Randomized clinical controlled trials } \\ \text { BMD: } & \text { Bone mineral density } \\ \text { VAS: } & \text { Visual analogue scale } \\ \text { PRISMA: } & \text { Preferred reporting item for systematic } \\ & \text { review and meta-analysis } \\ \text { CNKI: } & \text { China National Knowledge Infrastructure } \\ \text { VIP: } & \text { Chinese Scientific Journal Database } \\ \text { CBM: } & \text { China Biology Medicine } \\ \text { MD: } & \text { Mean difference } \\ \text { SMD: } & \text { Standardized mean difference } \\ \text { RR: } & \text { Risk ratio } \\ \text { 95\% CI: } & \text { 95\% confidence interval } \\ \text { GRADE: } & \text { Grading of Recommendations Assessment, } \\ \text { ICA: } & \text { Development, and Evaluation } \\ \text { OA: } & \text { Icariin } \\ \text { OVX: } & \text { Oleanolic acid } \\ \text { HRT: } & \text { Ovariectomization } \\ & \text { Hormone replacement therapy. }\end{array}$

\section{Data Availability}

The data used to support the findings of this study have been deposited in the following repository: PubMed: https://www. ncbi.nlm.nih.gov/pubmed/; Cochrane Library: https://www. cochranelibrary.com/; EMBASE: https://www.embase.com/; Web of Science: http://webofscience.com/; CNKI: https:// www.cnki.net/; VIP: http://www.cqvip.com/; Wanfang: http://www.wanfangdata.com.cn/; CBM: http://www. sinomed.ac.cn/.

\section{Conflicts of Interest}

The authors have no conflicts of interest to disclose. 


\section{Authors' Contributions}

Junquan Liang, Guizhen Chen, and Yunxiang Xu conceptualized the study and were responsible for writing-review and editing. Yunxiang $\mathrm{Xu}$ and Junquan Liang curated the data. Junquan Liang and Fengyi Wang performed formal analysis and were responsible for methodology, resources, software, visualization, and writing-original draft. Guizhen Chen and Yunxiang $\mathrm{Xu}$ were responsible for project administration.

\section{Acknowledgments}

This work was supported by the National Natural Science Foundation of China (grant no. 81574064, Yunxiang Xu; 81473755, Guizhen Chen); the Shenzhen Science and Technology Planning Project (grant no. JCYJ20170306152650625); the Shenzhen Bao'an District TCM Hospital Research Program (no. BAZYY20200609).

\section{References}

[1] C. D. Wylie, "Setting a standard for a "silent" disease: defining osteoporosis in the 1980s and 1990s," Studies in History and Philosophy of Science Part C: Studies in History and Philosophy of Biological and Biomedical Sciences, vol. 41, no. 4, pp. 376-385, 2010.

[2] H. Orimo, Y. Sugioka, M. Fukunaga et al., "Diagnostic criteria of primary osteoporosis," Journal of Bone and Mineral Metabolism, vol. 16, no. 3, pp. 139-150, 1998.

[3] E. S. Muto, R. Adler, J. Bilezikian et al., "The clinical diagnosis of osteoporosis: a position statement from the national bone health alliance working group," Osteoporosis International, vol. 25, no. 5, pp. 1439-1443, 2014.

[4] F. Bolognese and W. Xia, "The epidemiology of osteoporosis, associated fragility fractures, and management gap in China," Arch Osteoporos, vol. 14, no. 1, p. 32, 2019.

[5] A. Odén, E. V. McCloskey, J. A. Kanis, N. C. Harvey, and H. Johansson, "Burden of high fracture probability worldwide: secular increases 2010-2040," Osteoporosis International, vol. 26, no. 9, pp. 2243-2248, 2015.

[6] L.-Q. Yuan, X. Lin, D. Xiong et al., "Epidemiology and management of osteoporosis in the People's Republic of China: current perspectives," Clinical Interventions in Aging, vol. 10, pp. 1017-1033, 2015.

[7] Y. Wang, Y. Tao, M. E. Hyman, J. Li, and Y. Chen, "Osteoporosis in China," Osteoporosis International, vol. 20, no. 10, pp. 1651-1662, 2009.

[8] T. Chandran and I. Venkatachalam, "Efficacy and safety of denosumab compared to bisphosphonates in improving bone strength in postmenopausal osteoporosis: a systematic review," Singapore Medical Journal, vol. 60, no. 7, pp. 364-378, 2019.

[9] S. D. Berry, S. Shi, and D. P. Kiel, "Considering the risks and benefits of osteoporosis treatment in older adults," JAMA Internal Medicine, vol. 179, no. 8, pp. 1103-1104, 2019.

[10] D. M. Black and C. J. Rosen, "Postmenopausal osteoporosis," New England Journal of Medicine, vol. 374, no. 3, pp. 254-262, 2016.

[11] M. K. Skjødt, M. Frost, and B. Abrahamsen, "Side effects of drugs for osteoporosis and metastatic bone disease," British Journal of Clinical Pharmacology, vol. 85, no. 6, pp. 1063-1071, 2019.
[12] Q. Zeng, N. Li, Q. Wang et al., "The prevalence of osteoporosis in china, a nationwide, multicenter DXA survey," Journal of Bone and Mineral Research, vol. 34, no. 10, pp. 1789-1797, 2019.

[13] P.-C. Leung and W.-S. Siu, "Herbal treatment for osteoporosis: a current review," Journal of Traditional and Complementary Medicine, vol. 3, no. 2, pp. 82-87, 2013.

[14] Y.-M. Xie, Y. Yuwen, F.-H. Dong et al., "Clinical practice guideline of traditional medicine for primary osteoporosis," Chinese Journal of Integrative Medicine, vol. 17, no. 1, pp. 52-63, 2011.

[15] J.-B. Sun, M.-H. Chen, and D.-K. Lin, "New insights into the tonifying kidney-yin herbs and formulas for the treatment of osteoporosis," Archives of Osteoporosis, vol. 12, no. 1, p. 14, 2017.

[16] B. Shu, Q. Shi, and Y.-J. Wang, "Shen (kidney)-tonifying principle for primary osteoporosis: to treat both the disease and the Chinese medicine syndrome," Chinese Journal of Integrative Medicine, vol. 21, no. 9, pp. 656-661, 2015.

[17] H. Lin, X. Wang, Y. Mo et al., "Acupuncture for primary osteoporosis: evidence, potential treatment prescriptions, and mechanisms," Evidence-Based Complementary and Alternative Medicine, vol. 2019, Article ID 2705263, 15 pages, 2019.

[18] D. Atkins, D. Best, P. A. Briss et al., "Grading quality of evidence and strength of recommendations," BMJ (Clinical Research Ed), vol. 328, no. 7454, p. 1490, 2004.

[19] Y. X. Zheng, X. Z. Wang, and Z. K. Zhang, "Bushen Yijing Fang reduces fall risk in late postmenopausal women with osteopenia: a randomized double-blind and placebo-controlled trial," Scientific Reports, vol. 9, p. 2089, 2019.

[20] H. M. Zhu, L. Qin, P. Garnero et al., “The first multicenter and randomized clinical trial of herbal Fufang for treatment of postmenopausal osteoporosis," Osteoporosis International, vol. 23, no. 4, pp. 1317-1327, 2012.

[21] Z. Q. Zhao and X. X. Yan, "A study on the improvement of clinical symptoms of primary osteoporosis with tonifying kidney method by traditional Chinese medicine," Chinese Journal of Osteoporosis, vol. 24, no. 3, pp. 371-375, 2018.

[22] Y. Yuan, T. Tang, S. Q. Shi, and W. D. Wang, "Clinical application effect of therapy combined traditional Chinese and western medicine for senile osteoporosis," Chinese Archives of Traditional Chinese Medicine, vol. 32, no. 8, pp. 2014-2017, 2014.

[23] P. Huang, "Observation on the curative effect of Chinese and Western medicine in the treatment of primary osteoporosis," Journal of Practical Traditional Chinese Medicine, vol. 31, no. 4, Article ID 2015530819, 298-299 pages, 2015.

[24] W. Wu, D. F. Li, X. Zhi, and H. Meiqing, "Preventive and therapeutic effects of xianling gubao capsules for postmenopausal osteoporosis," Journal of Guangzhou University of Traditional Chinese Medicine, vol. 22, no. 3, Article ID 2005276556, 191-193 pages, 2005.

[25] B. X. Liu, L. P. Wang, M. Xu et al., "Postmenopausal osteoporosis of liver and kidney deficiency type treated with acupoint catgut embedding by stages," Chinese Acupuncture \& Moxibustion, vol. 31, no. 4, pp. 315-319, 2011.

[26] B. X. Liu, L. P. Wang, M. Xu, C. J. Huang, and F. Y. Tang, "Effect of embedding thread at acupoints on TCM syndrome integral and quality of life scale of the patients with postmenopausal osteoporosis of liver and kidney deficiency type," Chinese Archives of Traditional Chinese Medicine, vol. 29, no. 3, pp. 550-552, 2011.

[27] Y. J. Qi, "Clinical effect of bushen qianggu decoction on senile osteoporosis," Modern Journal of Integrated Traditional 
Chinese and Western Medicine, vol. 25, no. 22, Article ID 2016515893, 2461-2463 pages, 2016.

[28] Y. D. Li, Z. Q. Fan, and C. Y. Lu, "The effect of Bushen Jianpi Decoction assisted with western medicine on senile osteoporosis and its effect on bone mineral density," Nei Mongol Journal of Traditional Chinese Medicine, vol. 36, no. 21, pp. 105-106, 2017.

[29] J. Hu and C. Li, "Observation on the therapeutic effect of Tonifying the kidney and strengthening the spleen on primary osteoporosis," Hubei Journal of Traditional Chinese Medicine, vol. 34, no. 4, Article ID 2012349754, 11-12 pages, 2012.

[30] W. X. Liu and Y. Y. Gong, "Study on the therapeutic effect of Bushen Jianpi Huoxue decoction combined with alendronate sodium tablets on osteoporosis," Shaanxi Journal of Traditional Chinese Medicine, vol. 39, no. 10, pp. 1426-1428, 2018.

[31] S. Chen, L. Xie, Y. Tao, G. Xu, and Y. M. Ma, "Observation on the therapeutic effect of Bushen Jiangu formula combined with salmon calcitonin on senile osteoporosis," Chinese Journal of Traditional Medical Science and Technology, vol. 24, no. 3, pp. 320-321, 2017.

[32] Z. Zou, "Clinical effect of bushen jiangu decoction on postmenopausal osteoporosis," Journal of Modern Medicine \& Health, vol. 32, no. 11, pp. 1725-1726, 2016.

[33] J. Han, "Primary osteoporosis randomized parallel group study bushenzhuanggutang combined western medicine," Journal of Practical Traditional Chinese Internal Medicine, vol. 12, pp. 32-34, 2013.

[34] Y. Q. Huang, M. Shao, S. J. Xu, and T. Jiang, "Clinical observation on bushen tang combined with alendronate sodium tablets for primary osteoporosis with kidney deficiency type," Journal of New Chinese Medicine, vol. 50, no. 9, pp. 102-105, 2018.

[35] S. Huang, J. Y. Chen, and H. Gao, "The effect of bushen huoxue recipe on bone density and the biochemical index of bone metabolism in senile osteoporosis," Journal of Clinical Orthopaedics, vol. 20, no. 5, pp. 629-632, 2017.

[36] S. Song, C. Liu, and D. L. Jiang, "Random parallel control shudy of bushen huoxue recipe combine with miacalcic on senile osteoporosis," Journal of Practical Traditional Chinese Internal Medicine, vol. 29, no. 5, pp. 88-90, 2015.

[37] J. Pan and W. T. Ding, "Study on the clinical effect of Bushen Huoxue decoction combined with oyster calcium carbonate in the treatment of primary osteoporosis of middle-aged and old people in rural areas," Chinese Journal of Clinical Rational Drug Use, vol. 33, pp. 133-134, 2014.

[38] X. W. Wang, "Clinical observation on 100 cases of postmenopausal primary osteoporosis treated with Bushen Huoxue Decoction and calcium Erqi," Chinese Journal of Ethnomedicine and Ethnopharmacy, vol. 27, no. 24, pp. 127129, 2018.

[39] X. Gui, G. X. Gong, and F. M. Huang, "Clinical observation of 60 cases of primary osteoporosis treated with bushen yiqi huayu decoction," Drugs and Clinic, vol. 14, no. 17, Article ID 2017703424, 18-20 pages, 2017.

[40] B. X. Liu, C. J. Huang, D. B. Liang, and H. W. Tang, "Clinical control study on postmenopausal osteoporosis treated with embedding thread according to syndrome differentiation and medication," Chinese Journal of Integrated Traditional and Western Medicine, vol. 31, no. 10, pp. 1349-1354, 2011.

[41] X. Y. Xie, W. J. Zhang, W. W. Yu, and X. H. Yang, "Effect of gushen recipe on bone turnover in osteoporosis patients," Journal of Guangzhou University of Traditional Chinese Medicine, vol. 35, no. 5, pp. 805-809, 2018.
[42] Y. Liu and C. W. Wang, "Clinical effect of Erxian Bushen decoction combined with warm acupuncture in treating postmenopause osteoporosis with syndrome of kidney-Yang deficiency," Chinese Journal of Experimental Traditional Medical Formulae [zhongguo Shi Yan Fang Ji Xue Za Zhi], vol. 22, no. 9, pp. 162-166, 2016.

[43] D.-M. Xu, H. Xu, J. Liu et al., "of thunder-fire moxibustion on pain, quality of life, and tension of multifidus in patients with primary osteoporosis: a randomized controlled trial," Medical Science Monitor, vol. 24, pp. 2937-2945, 2018.

[44] X. L. Han, Z. J. Lou, and H. W. Lin, "Treatment of senile osteoporosis with shugan wenshen tanyu shuangjie decoction and acupuncture," Gansu Journal of Traditional Chinese Medicine, vol. 24, no. 2, pp. 32-34, 2011.

[45] D. N. Ma and Y. J. Fan, "Study on the effect of combination of traditional Chinese medicine and acupuncture in the treatment of osteoporosis," Contemporary Medical Symposium, vol. 16, no. 7, pp. 58-59, 2018.

[46] G. O. Ouyang, D.-Y. Wang, and X.-M. Xu, "Clinical observation on acupuncture for male osteoporosis," World Journal of Acupuncture-Moxibustion, vol. 31, no. 1, pp. 23-25, 2011.

[47] S. J. Lu, "Effective observation on treating postmenopausal osteoporosis by catgut embedding therapy combined with medicine," Clinical Journal of Chinese Medicine, vol. 6, no. 11, pp. 48-49, 2014.

[48] N. J. Peng, P. C. Zhong, and X. H. Zhao, "Clinical observation on 35 cases of primary osteoporosis treated by Acupoint Catgut Embedding," Hunan Journal of Traditional Chinese Medicine, vol. 33, no. 10, pp. 101-102, 2017.

[49] N. J. Peng, P. C. Zhong, and X. H. Zhao, "Clinical study of acupoint catgut-embedding on primary osteoporosis," Guiding Journal of Traditional Chinese Medicine and Pharmacy, vol. 24, no. 12, pp. 72-74, 2018.

[50] P. C. Zhong, N. J. Peng, and X. H. Zhao, "Observation of the curative effect of acupoint catgut embedding combined with western medicine on osteoporosis," Modern Journal of Integrated Traditional Chinese and Western Medicine, vol. 27, no. 16, pp. 1714-1716, 2018.

[51] Z. W. Lin, "The effect of Shenshu acupoint catgut embedding on bone mineral density of primary osteoporosis," Chinese Journal of Osteoporosis, vol. 12, no. 4, pp. 381-383, 2006.

[52] Z. W. Lin, "Clinical study on Shenshu acupoint catgut embedding therapy for postmenopausal osteoporosis," Journal of Clinical Acupuncture and Moxibustion, vol. 22, no. 4, pp. 1-3, 2006.

[53] X. J. Zou, Y. Y. Hao, J. Hou, and S. L. Pan, "Curative effect observation on point injection of saline combined with routine western medicine for primary senile osteoporosis," Journal of New Chinese Medicine, vol. 50, no. 11, pp. 183-185, 2018.

[54] Z. H. Zhou, N. Q. Wang, C. E. Ting, X. G. Zhou, and J. W. Zhou, "Postmenopausal osteoporosis treated with acupoint injection of salmon calcitonin:a randomized contro," Chinese Acupuncture \& Moxibustion, vol. 36, no. 7, pp. 705-708, 2016.

[55] X. Y. Zheng, Clinical Research Guidance of New Chinese Herbal Medicine (China), China Medical Science and Technology Press, 356-360 pages Beijing, China, 2002.

[56] S.-B. Su, W. Jia, A. Lu, and S. Li, "Evidence-based ZHENG: a traditional Chinese medicine syndrome," Evidence-based Complementary and Alternative Medicine: ECAM, vol. 2014, Article ID 484201, 2 pages, 2014.

[57] Z. Gao, Y. Lu, U. Halmurat-Upur, J. Jing, and D. Xu, "Study of osteoporosis treatment principles used historically by ancient 
physicians in Chinese medicine," Chinese Journal of Integrative Medicine, vol. 19, no. 11, pp. 862-868, 2013.

[58] Y. Liu, J. P. Liu, and Y. Xia, "Chinese herbal medicines for treating osteoporosis," Cochrane Database of Systematic Reviews, vol. 3, Article ID CD005467, 2014.

[59] W.-M. Deng, P. Zhang, H. Huang et al., "Five-year follow-up study of a kidney-tonifying herbal Fufang for prevention of postmenopausal osteoporosis and fragility fractures," Journal of Bone and Mineral Metabolism, vol. 30, no. 5, pp. 517-524, 2012.

[60] Y. Shen, X. Liu, H. Li, H. Liu, Z. Pan, and G. Chen, "Brain neural effects of the 'tonifying kidney and benefiting marrow' method in the treatment of osteoporosis," Journal of Traditional Chinese Medicine, vol. 39, no. 6, pp. 902-909, 2019.

[61] Q. Bian, J. H. Huang, S. F. Liu et al., "Different molecular targets of Icariin on bMSCs in CORT and OVX -rats," Frontiers in Bioscience (Elite Edition), vol. 4, pp. 1224-1236, 2012.

[62] H. Wang, M.-H. Ma, S.-S. Nian, and L.-L. Xu, "Antiosteoporotic activity of icariin in ovariectomized rats," Phytomedicine, vol. 16, no. 4, pp. 320-326, 2009.

[63] J.-F. Li, Y. Zhao, M.-M. Cai, X.-F. Li, and J.-X. Li, "Synthesis and evaluation of a novel series of heterocyclic oleanolic acid derivatives with anti-osteoclast formation activity," European Journal of Medicinal Chemistry, vol. 44, no. 7, pp. 2796-2806, 2009.

[64] Z. Yang, J. H. Huang, and S. F. Liu, “The osteoprotective effect of psoralen in ovariectomy-induced osteoporotic rats via stimulating the osteoblastic differentiation from bone mesenchymal stem cells," Menopause, vol. 19, no. 10, pp. 1156-1164, 2012.

[65] Y. Zhao, Y. Huai, J. Jin, M. Geng, and J.-X. Li, “Quinoxaline derivative of oleanolic acid inhibits osteoclastic bone resorption and prevents ovariectomy-induced bone loss," Menopause, vol. 18, no. 6, pp. 690-697, 2011.

[66] X. Zheng, G. Wu, Y. Nie, and Y. Lin, "Electroacupuncture at the governor vessel and bladder meridian acupoints improves postmenopausal osteoporosis through osteoprotegerin/ RANKL/RANK and Wnt/ $\beta$-catenin signaling pathways," Experimental and Therapeutic Medicine, vol. 10, no. 2, pp. 541-548, 2015.

[67] G.-Z. Chen, Y.-X. Xu, J.-W. Zhang, S.-H. Liu, and Z.-Y. Guo, "Effect of acupoint catgut-embedding on the quality of life, reproductive endocrine and bone metabolism of postmenopausal women," Chinese Journal of Integrative Medicine, vol. 16, no. 6, pp. 498-503, 2010.

[68] L. Zhang, Y. Wang, Y. Xu et al., "Metabonomic analysis reveals efficient ameliorating effects of acupoint stimulations on the menopause-caused alterations in mammalian metabolism," Scientific Reports, vol. 4, p. 3641, 2014. 